\title{
Non-Abelian, supersymmetric black holes and strings in 5 dimensions
}

\author{
Patrick Meessen, ${ }^{b}$ Tomás Ortín ${ }^{a}$ and Pedro F. Ramírez ${ }^{a}$ \\ ${ }^{a}$ Instituto de Física Teórica UAM/CSIC, \\ C/ Nicolás Cabrera, 13-15, C.U. Cantoblanco, E-28049 Madrid, Spain \\ ${ }^{b}$ HEP Theory Group, Departamento de Física, Universidad de Oviedo, \\ Avda. Calvo Sotelo s/n, E-33007 Oviedo, Spain \\ E-mail: meessenpatrick@uniovi.es, Tomas.Ortin@csic.es, \\ p.f.ramirez@csic.es
}

ABSTRACT: We construct and study the first supersymmetric black-hole and black-string solutions of non-Abelian-gauged $\mathcal{N}=1, d=5$ supergravity $(\mathcal{N}=1, d=5$ Super-EinsteinYang-Mills theory) with non-trivial SU(2) gauge fields: BPST instantons for black holes and BPS monopoles of different kinds ('t Hooft-Polyakov, Wu-Yang and Protogenov) for black strings and also for certain black holes that are well defined solutions only for very specific values of all the moduli. Instantons, as well as colored monopoles do not contribute to the masses and tensions but do contribute to the entropies.

The construction is based on the characterization of the supersymmetric solutions of gauged $\mathcal{N}=1, d=5$ supergravity coupled to vector multiplets achieved in ref. [1] which we elaborate upon by finding the rules to construct supersymmetric solutions with one additional isometry, both for the timelike and null classes. These rules automatically connect the timelike and null non-Abelian supersymmetric solutions of $\mathcal{N}=1, d=5 \mathrm{SEYM}$ theory with the timelike ones of $\mathcal{N}=2, d=4$ SEYM theory $[2,3]$ by dimensional reduction and oxidation. In the timelike-to-timelike case the singular Kronheimer reduction recently studied in ref. [4] plays a crucial role.

KEYwords: Black Holes, Supergravity Models, Black Holes in String Theory

ArXiv EPrint: 1512.07131 


\section{Contents}

1 Introduction 1

$2 \mathcal{N}=1, d=5$ SEYM theories $\quad 4$

3 The supersymmetric solutions of $\mathcal{N}=1, d=5$ SEYM theories $\quad 8$

3.1 Timelike supersymmetric solutions 8

3.1.1 Timelike supersymmetric solutions with one isometry 10

3.1.2 Dimensional reduction of the timelike supersymmetric solutions with one isometry 12

$\begin{array}{lll}3.2 & \text { Null supersymmetric solutions } & 13\end{array}$

3.2.1 $u$-independent null supersymmetric solutions 13

3.2.2 Dimensional reduction of the $u$-independent null supersymmetric solutions $\quad 15$

4 5-dimensional supersymmetric non-Abelian solutions of the SU(2)$\begin{array}{lr}\text { gauged ST }[2,5] \text { model } & 16\end{array}$

$\begin{array}{ll}4.1 \text { The models } & 16\end{array}$

$\begin{array}{ll}4.2 \text { The solutions } & 18\end{array}$

4.2.1 A simple 5d black hole with non-Abelian hair $\quad 18$

4.2.2 A rotating $5 d$ black hole with non-Abelian hair 20

$\begin{array}{lll}4.2 .3 & \text { A more general solution } & 21\end{array}$

4.2.4 Null supersymmetric non-Abelian $5 d$ solutions from $4 d$ black holes and global monopoles $\quad 23$

$\begin{array}{lll}5 & \text { Conclusions } & 25\end{array}$

A Dimensional reduction of $\mathcal{N}=1, d=5$ SEYM theories $\quad 26$

B Spherically-symmetric solutions of the SU(2) Bogomol'nyi equations in $\begin{array}{ll}\mathbb{E}^{3} & \mathbf{2 7}\end{array}$

\section{Introduction}

The search for classical solutions of General Relativity and theories of gravity in general has proven to be one of the most fruitful approaches to study this universal and mysterious interaction. This is partially due to the non-perturbative information they provide, which we do not know how to obtain otherwise. It is fair to say that some of the solutions discovered (such as the Schwarzschild and Kerr black-hole solutions, the cosmological ones or the $\mathrm{AdS}_{5} \times \mathrm{S}^{5}$ solution of type IIB supergravity) have opened entire fields of research. 
Some of the most interesting solutions are supported by fundamental matter fields and a large part of the search for gravity solutions has been carried out in theories in which gravity is coupled to different forms of matter, usually scalar fields, Abelian vector and $p$-form fields coupled in gauge-invariant ways among themselves and to scalars, as suggested by superstring and supergravity theories, for instance. The solutions of gravity coupled to non-Abelian vector fields have been much less studied because of the complexity of the equations. Most of the genuinely non-Abelian solutions found so far, such as the Bartnik-McKinnon particle [5] and its black hole-type generalizations [6], in the $\mathrm{SU}(2)$ Einstein-Yang-Mills (EYM) theory, are only known numerically, which makes them more difficult to study and generalize.

Supersymmetry can simplify dramatically the construction of classical solutions, providing in some cases recipes to construct systematically whole families of solutions that have the property of being "supersymmetric" or "having unbroken supersymmetry", or being "BPS" (a much less precise term) because these solutions satisfy much easier to solve first-order differential equations. ${ }^{1}$ These techniques can be applied to non-supersymmetric theories if we can "embed" them in a larger supersymmetric theory from which they can be obtained by a consistent truncation that, in particular, gets rid of the fermionic fields.

In order to apply these techniques to the case of theories of gravity coupled to fundamental matter fields we must embed the theories first in supergravity theories. $d=4$ EYM theories can be embedded almost trivially in $\mathcal{N}=1, d=4$ gauged supergravity coupled to vector supermultiplets, but there are no supersymmetric black-hole or more general particle-like solutions in $\mathcal{N}=1, d=4$ supergravity: all the supersymmetric solutions of these theories belong to the null class ${ }^{2}$ and describe, generically, massless solutions such as gravitational waves and also black strings (whose tension does not count as a mass). This could well explain why there are no simple analytic solutions of the EYM theory.

Embedding of $d=4$ EYM theories in extended $(\mathcal{N}>1) d=4$ supergravity theories turns out to be impossible, since the latter always include additional scalar fields charged under the non-Abelian fields which cannot be consistently truncated away. On the other hand, these scalar fields (or part of them) can also be interpreted as Higgs fields and we can think of those supergravities (which we will call Super-Einstein-Yang-Mills (SEYM) theories) as the minimal supersymmetric generalizations of the Einstein-Yang-Mills-Higgs (EYMH) theory. Actually, some solutions of the SEYM theories are also solutions of the EYMH theory, but this is not generically true and we cannot say that the EYMH theory is embedded in some SEYM theory.

At any rate, analytic supersymmetric solutions of SEYM or more general gauged supergravity theories should be much easier to find than solutions of the EYM theory and, at the same time, much more realistic, since we know there are scalar fields charged under non-Abelian vector fields in Nature.

This expectation turns out to be true. In 1991 Harvey and Liu [8] and in 1997 Chamseddine and Volkov $[9,10]$ found globally regular gravitating monopole ("global monopole")

\footnotetext{
${ }^{1}$ For a general review on the construction of supersymmetric solutions of supergravity theories, including some of those that we are going to study here, see ref. [7].

${ }^{2}$ The Killing spinor of the supersymmetric solutions in the null (resp. timelike) class gives rise to a null (resp. timelike) Killing vector bilinear.
} 
solutions to gauged $\mathcal{N}=4, d=4$ supergravity, a theory that can be related to the Heterotic string. In 1994, a 4-dimensional black-hole solution with non-Abelian hair was obtained by adding stringy (Heterotic) $\alpha^{\prime}$ corrections to an $a=1$ dilaton black hole [11]. This solution was singular in the Einstein frame. ${ }^{3}$ More recently, the timelike supersymmetric solutions of gauged $\mathcal{N}=2, d=4$ and $\mathcal{N}=1, d=5$ were characterized, respectively, in refs. $[2,13]$ and $[1,14],{ }^{4}$ so the form of all the fields in those solutions is given in terms of a few functions that satisfy first-order equations.

In the 4-dimensional case, these first-order equations are straightforward generalizations of the well-known Bogomol'nyi monopole equations [15] whose more general static and spherically symmetric solutions for the gauge group $\mathrm{SU}(2)$ were obtained by Protogenov in ref. [16]. Then, the characterization of timelike supersymmetric solutions was immediately used to construct, apart from global monopole solutions, the first analytical, regular, static, non-Abelian black-hole solutions which cannot be considered as pure Abelian embeddings [2], showing how the attractor mechanism works in the non-Abelian setting [2, 3]. Colored black holes ${ }^{5}$ and two-center non-Abelian solutions were constructed, respectively, in [17] and [12] by using, respectively, "colored monopole" and two-center solutions of the Bogomol'nyi equations.

In the $\mathcal{N}=1, d=5$ SEYM case, the characterization obtained in refs. $[1,14]$ has not yet been exploited. Doing so to construct non-Abelian black-hole and black-string solutions is our main goal in this paper. It is a well-known fact, one that also holds in the Abelian (ungauged) case, that the vector field strengths of the timelike supersymmetric solutions of these theories are the sum of two pieces, one of them self-dual in the hyperKähler base space, i.e. an instanton in the base space. In the non-Abelian case we are interested in, this fact can be exploited in an obvious way to add non-Abelian hair to black hole solutions.

As we are going to see, it will be convenient to refine the general characterization obtained in those references to obtain a simpler recipe to construct supersymmetric solutions with one additional isometry. These solutions are still general enough and can also be related to the timelike supersymmetric solutions of $\mathcal{N}=2, d=4$ SEYM. In the timeliketo-timelike reduction, we recover the relation between self-dual instantons in hyperKähler spaces with one isometry and BPS monopoles in $\mathbb{E}^{3}$ found by Kronheimer in ref. [18]. As we have shown in ref. [4] this redox relation brings us from singular colored monopoles to globally regular BPST instantons and vice-versa and it will allow us to obtain regular black holes with a BPST instanton field.

The recipes we have obtained can be applied to any model of $\mathcal{N}=1, d=5$ supergravity coupled to vector multiplets in which a non-Abelian subgroup of the perturbative duality group can be gauged. The explicit solutions we will construct will belong to a particular model, the ST $[2,5]$ model which is the smallest of the ST $[2, n]$ family of models admitting a $\mathrm{SU}(2)$ gauging. These models are consistent truncations of $\mathcal{N}=1, d=10$

\footnotetext{
${ }^{3}$ We will see, though, that it is closely related to the 4-dimensional black-hole solutions studied in [12] and to the 5-dimensional ones presented here.

${ }^{4}$ In the $\mathcal{N}=1, d=5$ case, the null supersymmetric solutions were characterized as well.

${ }^{5}$ Colored black holes have non-Abelian hair but vanishing asymptotic charges. The charges must be screened at infinity because they contribute to the near-horizon geometry and to the entropy.
} 
supergravity coupled to a number of vector multiplets on $T^{5}$ and, for low values of $n$, they can be embedded in Heterotic string theory. The $\mathrm{SU}(2)$ gauging can be associated to the enhancement of symmetry at the self-dual radius $\mathrm{U}(1) \times \mathrm{U}(1) \rightarrow \mathrm{U}(1) \times \mathrm{SU}(2)$, although, in order to study the details of the embedding of our model in Heterotic string theory (which will be our next goal) more work will be necessary.

This paper is organized as follows: in section 2 we review the gauging of a nonAbelian group of isometries of an $\mathcal{N}=1, d=5$ supergravity theory coupled to vector multiplets. The result of this procedure is what we call an $\mathcal{N}=1, d=5$ Super-EinsteinYang-Mills (SEYM) theory. In section 3 we review and extend the results of ref. [1] on the characterization of the supersymmetric solutions of $\mathcal{N}=1, d=5$ SEYM theories, giving the recipe to construct those admitting additional isometries and showing how they are related to the analogous supersymmetric solutions of $\mathcal{N}=2, d=4$ SEYM theories characterized in ref. $[3,13]$. We will then use these results in section 4 to construct black holes and black strings (in the timelike and null cases, respectively) of the $\mathrm{SU}(2)$-gauged ST[2,5] model of $\mathcal{N}=1, d=5$ supergravity and to study their relations, via dimensional reduction, to the non-Abelian timelike supersymmetric solutions (black holes and global monopoles) of the $\mathrm{SU}(2)$-gauged ST[2,5] model of $\mathcal{N}=2, d=4$ supergravity (see ref. [12]). Our conclusions are given in section 5. Appendix A reviews the reduction of ungauged $\mathcal{N}=1, d=5$ supergravity to a cubic model of $\mathcal{N}=2, d=4$ supergravity, with the relation between the 5- and 4-dimensional fields for any kind of solution (supersymmetric or not). This relation remains true for gauged supergravity theories under standard dimensional reduction (which does not change the gauge group). Finally, appendix B review the spherically-symmetric solutions of the Bogomol'nyi equation in $\mathbb{E}^{3}$ for $\mathrm{SU}(2)$.

\section{$2 \mathcal{N}=1, d=5$ SEYM theories}

In this section we give a brief description of general $\mathcal{N}=1, d=5$ Super-Einstein-Yang-Mills (SEYM) theories. These are theories of $\mathcal{N}=1, d=5$ supergravity coupled to $n_{v}$ vector supermultiplets (no hypermultiplets) in which a necessarily non-Abelian group of isometries of the Real Special manifold has been gauged. These theories can be considered the simplest supersymmetrization of non-Abelian Einstein-Yang-Mills theories in $d=5$. Our conventions are those in refs. $[1,19]$ which are those of ref. [20] with minor modifications.

The supergravity multiplet is constituted by the graviton $e^{a}{ }_{\mu}$, the gravitino $\psi_{\mu}^{i}$ and the graviphoton $A_{\mu}$. All the spinors are symplectic Majorana spinors and carry a fundamental $\mathrm{SU}(2) \mathrm{R}$-symmetry index. The $n_{v}$ vector multiplets, labeled by $x=1, \ldots, n_{v}$ consist of a real vector field $A_{\mu}^{x}$, a real scalar $\phi^{x}$ and a gaugino $\lambda^{i x}$.

The full theory is formally invariant under a $\mathrm{SO}\left(n_{v}+1\right)$ group $^{6}$ that mixes the matter vector fields $A^{x}{ }_{\mu}$ with the graviphoton $A_{\mu} \equiv A^{0}{ }_{\mu}$ and it is convenient to combine them into an $\mathrm{SO}\left(n_{v}+1\right)$ vector $\left(A^{I}{ }_{\mu}\right)=\left(A^{0}{ }_{\mu}, A^{x}{ }_{\mu}\right)$. It is also convenient to define a $\mathrm{SO}\left(n_{v}+1\right)$ vector of functions of the scalars $h^{I}(\phi)$. These $n_{v}+1$ functions of $n_{v}$ scalar must satisfy a

\footnotetext{
${ }^{6}$ The theory will only be invariant under a subgroup of $\mathrm{SO}\left(n_{v}+1\right)$.
} 
constraint. $\mathcal{N}=1, d=5$ supersymmetry determines that this constraint is of the form

$$
C_{I J K} h^{I}(\phi) h^{J}(\phi) h^{K}(\phi)=1,
$$

where the constant symmetric tensor $C_{I J K}$ completely characterizes the theory and the Special Real geometry of the scalar manifold. In particular, the kinetic matrix of the vector fields $a_{I J}(\phi)$ and the metric of the scalar manifold $g_{x y}(\phi)$ can be derived from it as follows: first, we define

$$
h_{I} \equiv C_{I J K} h^{J} h^{K}, \quad \Rightarrow \quad h^{I} h_{I}=1,
$$

and

$$
h_{x}^{I} \equiv-\sqrt{3} h_{, x}^{I} \equiv-\sqrt{3} \frac{\partial h^{I}}{\partial \phi^{x}}, \quad h_{I x} \equiv+\sqrt{3} h_{I, x}, \quad \Rightarrow \quad h_{I} h_{x}^{I}=h^{I} h_{I x}=0 .
$$

Then, $a_{I J}$ is defined implicitly by the relations

$$
h_{I}=a_{I J} h^{I}, \quad h_{I x}=a_{I J} h_{x}^{J} .
$$

It can be checked that

$$
a_{I J}=-2 C_{I J K} h^{K}+3 h_{I} h_{J} .
$$

The metric of the scalar manifold $g_{x y}(\phi)$, which we will use to raise and lower $x, y$ indices is (proportional to) the pullback of $a_{I J}$

$$
g_{x y} \equiv a_{I J} h_{x}^{I} h_{y}^{J}=-2 C_{I J K} h_{x}^{I} h_{y}^{J} h^{K} .
$$

The functions $h^{I}$ and their derivatives $h_{x}^{I}$ satisfy the following completeness relation:

$$
a_{I J}=h_{I} h_{J}+g_{x y} h_{I}^{x} h_{J}^{y}
$$

By assumption, the real Real Special structure is invariant under reparametrizations generated by vectors $k_{I}^{x}(\phi)^{7}$

$$
\delta \phi^{x}=c^{I} k_{I}^{x}
$$

satisfying the Lie algebra ${ }^{8}$

$$
\left[k_{I}, k_{J}\right]=-f_{I J}{ }^{K} k_{K} .
$$

The invariance of the metric $g_{x y}$ implies that the vectors $k_{I}^{x}(\phi)$ are Killing vectors. The invariance of the constraint eq. (2.1) implies the invariance of the $C_{I J K}$ tensor

$$
-3 f_{I(J}^{M} C_{K L) M}=0 .
$$

Multiplying this identity by $h^{J} h^{K} h^{L}$ we get another important relation:

$$
f_{I J}{ }^{K} h^{J} h_{K}=0 .
$$

\footnotetext{
${ }^{7}$ Some of these vectors may be identically zero. This is the price to be paid for labeling the gauge vectors and the Killing vectors with the same indices.

${ }^{8}$ Some of the structure constants may vanish identically, but it is assumed that some of them do not because, otherwise, we would be dealing with an ungauged supergravity.
} 
The functions $h^{I}(\phi)$, in their turn, must be invariant up to $\mathrm{SO}\left(n_{v}+1\right)$ rotations, that is

$$
k_{I}^{x} \partial_{x} h^{J}-f_{I K}^{J} h^{K}=0, \quad \Rightarrow \quad k_{I}^{x}=-\sqrt{3} f_{I J}^{K} h_{K}^{x} h^{J}, \quad \Rightarrow \quad h^{I} k_{I}^{x}=0 .
$$

If the real special manifold is a symmetric space, then the tensor $C_{I J K}$ satisfies the identity

$$
C^{I J K} C_{J(L M} C_{N P) K}=\frac{1}{27} \delta^{I}{ }_{(L} C_{M N P)},
$$

where $C^{I J K}=C_{I J K}$. In these spaces we can solve immediately $h^{I}$ in terms of the $h_{I}$

$$
h^{I}=27 C^{I J K} h_{J} h_{K}, \quad \Rightarrow \quad C^{I J K} h_{I} h_{J} h_{K}=\frac{1}{27} .
$$

To gauge this global symmetry group we promote the constant parameters $c^{I}$ to arbitrary spacetime functions identifying them with the gauge parameters of the vector fields $\Lambda^{I}(x) c^{I} \rightarrow-g \Lambda^{I}(x)$. The gauge transformations scalars $\phi^{x}$, the functions $h^{I}$ and the $A^{I}{ }_{\mu}$ take the form

$$
\begin{aligned}
\delta_{\Lambda} \phi^{x} & =-g \Lambda^{I} k_{I}^{x} \\
\delta_{\Lambda} h^{I} & =-g f_{J K}{ }^{I} \Lambda^{J} h^{K} \\
\delta_{\Lambda} A^{I}{ }_{\mu} & =\partial_{\mu} \Lambda^{I}+g f_{J K}{ }^{I} A^{J}{ }_{\mu} \Lambda^{K} \equiv \mathfrak{D}_{\mu} \Lambda^{I},
\end{aligned}
$$

where $\mathfrak{D}_{\mu}$ is the gauge-covariant derivative. $\mathfrak{D}_{\mu} h^{I}$ has the same expression as $\mathfrak{D}_{\mu} \Lambda^{I}$ and have the same gauge transformations as $h^{I}$ and $\Lambda^{I}$. We also have

$$
\begin{aligned}
\mathfrak{D}_{\mu} h_{I} & =\partial_{\mu} h_{I}+g f_{I J}{ }^{K} A^{J}{ }_{\mu} h_{K}, \\
\mathfrak{D}_{\mu} C_{I J K} & =0 .
\end{aligned}
$$

On the other hand, the gauge-covariant derivative of the scalars is given by

$$
\mathfrak{D}_{\mu} \phi^{x}=\partial_{\mu} \phi^{x}+g A^{I}{ }_{\mu} k_{I}^{x},
$$

and transforms as

$$
\delta_{\Lambda} \mathfrak{D}_{\mu} \phi^{x}=-g \Lambda^{I} \partial_{y} k_{I}^{x} \mathfrak{D}_{\mu} \phi^{x} .
$$

The gauginos $\lambda^{i x}$ transform in exactly the same way as $\mathfrak{D} \phi^{x}$ and their gauge-covariant derivatives are identical to the second covariant derivative of $\phi^{x}$ :

$$
\mathfrak{D}_{\mu} \mathfrak{D}_{\nu} \phi^{x}=\partial_{\mu} \mathfrak{D}_{\nu} \phi^{x}-\Gamma_{\mu \nu}^{\rho} \mathfrak{D}_{\rho} \phi^{x}+\Gamma_{y z}{ }^{x} \mathfrak{D}_{\mu} \phi^{y} \mathfrak{D}_{\nu} \phi^{z}+g A_{\mu}^{I} \partial_{y} k_{I}^{x} \mathfrak{D}_{\nu} \phi^{y} .
$$

The gauge-covariant vector field strength has the standard form

$$
F_{\mu \nu}^{I}=2 \partial_{[\mu} A_{\nu]}^{I}+g f_{J K}{ }^{I} A_{\mu}^{J} A_{\nu}^{K}{ }_{\nu}
$$

The bosonic action of $\mathcal{N}=1, d=5 \mathrm{SEYM}$ is given in terms of $a_{I J}, g_{x y}, C_{I J K}$ and the structure constants $f_{I J}{ }^{K}$ by

$$
S=\int d^{5} x \sqrt{g}\left\{R+\frac{1}{2} g_{x y} \mathfrak{D}_{\mu} \phi^{x} \mathfrak{D}^{\mu} \phi^{y}-\frac{1}{4} a_{I J} F^{I \mu \nu} F^{J}{ }_{\mu \nu}+\frac{1}{12 \sqrt{3}} C_{I J K} \frac{\varepsilon^{\mu \nu \rho \sigma \alpha}}{\sqrt{g}}\left[F^{I}{ }_{\mu \nu} F^{J}{ }_{\rho \sigma} A^{K}{ }_{\alpha}\right.\right.
$$




$$
\left.\left.-\frac{1}{2} g f_{L M}{ }^{I} F^{J}{ }_{\mu \nu} A^{K}{ }_{\rho} A^{L}{ }_{\sigma} A^{M}{ }_{\alpha}+\frac{1}{10} g^{2} f_{L M}{ }^{I} f_{N P}{ }^{J} A^{K}{ }_{\mu} A^{L}{ }_{\nu} A^{M}{ }_{\rho} A^{N}{ }_{\sigma} A^{P}{ }_{\alpha}\right]\right\} .
$$

Observe that this action does not contain a scalar potential $V(\phi)$ because

$$
V(\phi)=\frac{3}{2} g^{2} h^{I} h^{J} k_{I}^{x} k_{J}^{y} g_{x y},
$$

(the expression that follows from the general formula in ref. [20]) vanishes identically for the kind of gaugings considered here, owing to the property eq. (2.12). This fact is associated to the vanishing of the corresponding fermion shift in the gauginos' supersymmetry transformations.

The equations of motion for the bosonic fields are

$$
\begin{aligned}
\mathcal{E}_{\mu \nu} \equiv & \frac{1}{2 \sqrt{g}} e_{a(\mu} \frac{\delta S}{\delta e_{a}{ }^{\nu)}} \\
= & G_{\mu \nu}-\frac{1}{2} a_{I J}\left(F^{I}{ }_{\mu}{ }^{\rho} F^{J}{ }_{\nu \rho}-\frac{1}{4} g_{\mu \nu} F^{I \rho \sigma} F^{J}{ }_{\rho \sigma}\right) \\
& +\frac{1}{2} g_{x y}\left(\mathfrak{D}_{\mu} \phi^{x} \mathfrak{D}_{\nu} \phi^{y}-\frac{1}{2} g_{\mu \nu} \mathfrak{D}_{\rho} \phi^{x} \mathfrak{D}^{\rho} \phi^{y}\right) \\
\mathcal{E}_{I}{ }^{\mu} \equiv & \frac{1}{\sqrt{g}} \frac{\delta S}{\delta A^{I}{ }_{\mu}} \\
= & \mathfrak{D}_{\nu}\left(a_{I J} F^{J \nu \mu}\right)+\frac{1}{4 \sqrt{3}} \frac{\varepsilon^{\mu \nu \rho \sigma \alpha}}{\sqrt{g}} C_{I J K} F^{J}{ }_{\nu \rho} F^{k}{ }_{\sigma \alpha}+g k_{I x} \mathfrak{D}^{\mu} \phi^{x} \\
\mathcal{E}^{x} \equiv & -\frac{g^{x y}}{\sqrt{g}} \frac{\delta S}{\delta \phi^{y}} \\
= & \mathfrak{D}_{\mu} \mathfrak{D}^{\mu} \phi^{x}+\frac{1}{4} g^{x y} \partial_{y} a_{I J} F^{I \rho \sigma} F^{J}{ }_{\rho \sigma} .
\end{aligned}
$$

The supersymmetry transformation rules for the bosonic fields are

$$
\begin{aligned}
\delta_{\epsilon} e^{a}{ }_{\mu} & =\frac{i}{2} \bar{\epsilon}_{i} \gamma^{a} \psi_{\mu}^{i} \\
\delta_{\epsilon} A^{I}{ }_{\mu} & =-\frac{i \sqrt{3}}{2} h^{I} \bar{\epsilon}_{i} \psi_{\mu}^{i}+\frac{i}{2} h_{x}^{I} \bar{\epsilon}_{i} \gamma_{\mu} \lambda^{i x}, \\
\delta_{\epsilon} \phi^{x} & =\frac{i}{2} \bar{\epsilon}_{i} \lambda^{i x}
\end{aligned}
$$

and the corresponding transformation rules for the fermionic fields evaluated on vanishing fermions are

$$
\begin{aligned}
\delta_{\epsilon} \psi_{\mu}^{i} & =\nabla_{\mu} \epsilon^{i}-\frac{1}{8 \sqrt{3}} h_{I} F^{I \alpha \beta}\left(\gamma_{\mu \alpha \beta}-4 g_{\mu \alpha} \gamma_{\beta}\right) \epsilon^{i}, \\
\delta_{\epsilon} \lambda^{i x} & =\frac{1}{2}\left(\not \supset \phi^{x}-\frac{1}{2} h_{I}^{x} \not^{I}\right) \epsilon^{i},
\end{aligned}
$$

where $\nabla_{\mu} \epsilon^{i}$ is just the Lorentz-covariant derivative on the spinors, given in our conventions by

$$
\nabla_{\mu} \epsilon^{i}=\left(\partial_{\mu}-\frac{1}{4} \psi_{\mu}\right) \epsilon^{i}
$$


The equations of motion and the supersymmetry transformation rules are the straightforward covariantization of those of the ungauged theory, except for the addition of a source to the Maxwell equations corresponding to the charge carried by the scalar fields.

\section{The supersymmetric solutions of $\mathcal{N}=1, d=5$ SEYM theories}

In this section we are going to review first the results of ref. [1] particularized to the case in which there are no hypermultiplets nor Fayet-Iliopoulos terms. We will simply focus on the final characterization of the supersymmetric solutions. Then, we will analyze the form of the solutions that admit an additional isometry and can, therefore, be dimensionally reduced to $d=4$, following refs. [19, 21].

Let us start by reminding the reader that a solution of one of the $\mathcal{N}=1, d=5 \mathrm{SEYM}$ theories is said supersymmetric if the so-called Killing spinor equations

$$
\delta_{\epsilon} \psi_{\mu}^{i}=0, \quad \delta_{\epsilon} \lambda^{i x}=0,
$$

written in the background of the solution can be solved for at least one spinor $\epsilon^{i}(x)$, which is then called Killing spinor. The supersymmetric solutions of these theories can be classified according to the causal nature of the Killing vector that one can construct as a bilinear of the Killing spinor $V^{a}=i \bar{\epsilon}_{i} \gamma^{a} \epsilon^{i}$ as timelike $\left(V^{a} V_{a}>0\right)$ or null $\left(V^{a} V_{a}=0\right)$. These two cases must be discussed separately.

\subsection{Timelike supersymmetric solutions}

The fields of the timelike supersymmetric solutions of $\mathcal{N}=1, d=5$ SEYM theories are completely determined by

1. A choice of 4-dimensional (obviously Euclidean) hyperKähler metric

$$
d \hat{s}^{2}=h_{\underline{m n}}(x) d x^{m} d x^{n} .
$$

Fields and operators defined in this space are customarily hatted.

2. Vector fields defined in the hyperKähler space, $\hat{A}^{I}$, such that their 2-form field strengths, $\hat{F}^{I}(\hat{A})$ are self-dual

$$
\hat{\star} \hat{F}^{I}=+\hat{F}^{I},
$$

with respect to the hyperKähler metric. This implies that $\hat{A}^{I}$ defines an instanton solution of the Yang-Mills equations in the hyperKähler space.

3. A set of functions in the hyperKähler space $\hat{f}_{I}$ satisfying the equation ${ }^{9}$

$$
\hat{\mathfrak{D}}^{2} \hat{f}_{I}-\frac{1}{6} C_{I J K} \hat{F}^{J} \cdot \hat{F}^{K}=0 .
$$

Given $h_{\underline{m n}}, \hat{A}^{I}, \hat{f}_{I}$, the physical fields can be reconstructed as follows:

\footnotetext{
${ }^{9}$ The coefficient of the second term is wrong by a factor of 2 in refs. [1, 19] although all subsequent formulae are correct.
} 
1. The functions $\hat{f}_{I}$ are proportional to the $h_{I}(\phi)$ defined in eq. (2.2). The proportionality coefficient is called $1 / \hat{f}$ :

$$
h_{I} / \hat{f}=\hat{f}_{I} .
$$

The functions $h_{I}(\phi)$ satisfy a model-dependent constraint (analogous to the constraint satisfied by the functions $h^{I}(\phi)$, eq. (2.1)). This constraint can be obtained by solving eq. (2.2) for the $h^{I}$ and substituting the result into eq. (2.1). Therefore, the constraint has the form $F(h)=$.1 where $F$ is a function homogeneous of degree $3 / 2$ in the $h_{I}$ and, substituting the above equation, one gets

$$
\hat{f}^{-3 / 2}=F(\hat{f} .) .
$$

Using this result in eq. (3.5) one gets all the $h_{I}$ as in terms of the $\hat{f}_{I}$

$$
h_{I}=\hat{f}_{I} F^{-2 / 3}(\hat{f} .),
$$

and, using the expression of the $h^{I}$ in terms of the $h_{I}$, one also gets the $h^{I}$ in terms of the functions $\hat{f}_{I}$.

If the real special scalar manifold is symmetric, then we can use eq. (2.14) to get

$$
\hat{f}^{-3}=27 C^{I J K} \hat{f}_{I} \hat{f}_{J} \hat{f}_{K} .
$$

2. The scalar fields $\phi^{x}$ can be obtained by inverting the functions $h_{I}(\phi)$ or $h^{I}(\phi)$. A parametrization which is always available is

$$
\phi^{x}=h_{x} / h_{0}=\hat{f}_{x} / \hat{f}_{0} .
$$

3. Next, we define the 1 -form $\hat{\omega}$ through the equation

$$
(\hat{f} d \hat{\omega})^{+}=\frac{\sqrt{3}}{2} h_{I} \hat{F}^{I+} .
$$

4. Having solved the above equation for $\hat{\omega}$ we have determined completely the metric of the timelike supersymmetric solutions, which is given by

$$
d s^{2}=\hat{f}^{2}(d t+\hat{\omega})^{2}-\hat{f}^{-1} h_{\underline{m n}} d x^{m} d x^{n},
$$

5. Also, the complete 5 -dimensional vector fields are given by

$$
A^{I}=-\sqrt{3} h^{I} e^{0}+\hat{A}^{I}, \quad \text { where } \quad e^{0} \equiv \hat{f}(d t+\hat{\omega}),
$$

so that the spatial components are

$$
A^{I}{ }_{\underline{m}}=\hat{A}_{\underline{m}}^{I}-\sqrt{3} h^{I} \hat{f} \hat{\omega}_{\underline{m}} .
$$

The field strength can be written in the form

$$
F^{I}=-\sqrt{3} \hat{\mathfrak{D}}\left(h^{I} e^{0}\right)+\hat{F}^{I},
$$

where $\hat{\mathfrak{D}}$ is the covariant derivative in the hyperKähler space with connection $\hat{A}^{I}$. 


\subsubsection{Timelike supersymmetric solutions with one isometry}

We are particularly interested in the supersymmetric solutions that have an additional isometry. Following refs. [21, 22] we assume that the additional isometry is a triholomorphic isometry of the hyperKähler metric (i.e. an isometry respecting the hyperKähler structure), in which case, as shown in ref. [23] it must be a Gibbons-Hawking multi-instanton metric [24]. Assuming $z$ is the coordinate associated to the additional isometry, these metrics can always be written in the form

$$
h_{\underline{m n}} d x^{m} d x^{n}=H^{-1}(d z+\chi)^{2}+H d x^{r} d x^{r}, \quad r=1,2,3,
$$

where the $z$-independent function $H$ and 1 -form $\chi=\chi_{\underline{r}} d x^{r}$ are related by

$$
d \chi=\star_{3} d H,
$$

$\star_{3}$ being the Hodge operator in $\mathbb{E}^{3}$. Assuming now that the rest of the bosonic fields of the timelike supersymmetric solutions are $z$-independent one can simplify eqs. (3.3), (3.4) and (3.10).

Let us start with eq. (3.3) and let us assume that the selfduality of $\hat{F}^{I}$ has been defined with respect to the frame and orientation

$$
\hat{e}^{z}=H^{-1 / 2}(d z+\chi), \quad \hat{e}^{r}=H^{1 / 2} \delta^{r}{ }_{r} d x^{r}, \quad \varepsilon_{z 123}=+1 .
$$

Then, following Kronheimer [18], ${ }^{10}$ eq. (3.3) can be rewritten as Bogomol'nyi equations for a Yang-Mills-Higgs (YMH) system in the BPS limit in $\mathbb{E}^{3}[15]$

$$
\breve{\mathfrak{D}}_{r} \Phi^{I}=\frac{1}{2} \varepsilon_{r s t} \breve{F}^{I}{ }_{s t},
$$

where the 3-dimensional Higgs field and the vector fields are given by ${ }^{11}$

$$
\begin{aligned}
& 2 \sqrt{6} \Phi^{I} \equiv H \hat{A}_{\underline{z}}^{I}, \\
& 2 \sqrt{6} \breve{A}_{\underline{\underline{r}}}^{I} \equiv-\hat{A}_{\underline{r}}^{I}+\chi_{\underline{r}} \hat{A}_{\underline{z}}^{I} .
\end{aligned}
$$

Thus, we can always construct a selfdual YM instanton in a Gibbons-Hawking space from a (monopole) solution of the Bogomol'nyi equation of a YMH system in $\mathbb{E}^{3}$ $\left(\Phi^{I}, \breve{A}_{\underline{r}}^{I}\right)[18]$. Many solutions of these equations are known, specially in the spherically symmetric case. ${ }^{12}$ In ref. [4] this relation has been explored precisely for the SU(2) monopoles and instantons we are interested in, and we will make use of those results later.

We can now use this result into eq. (3.4), rewriting the 4-dimensional gauge vector in terms of the 3-dimensional gauge vector and Higgs field defined above and using the harmonicity of $H$ and the Bogomol'nyi equation to get rid of $\breve{F}^{I}$ and $\breve{\mathfrak{D}}^{2} \Phi^{I}$ (which vanishes identically). The result is the equation in $\mathbb{E}^{3}$

$$
\breve{\mathfrak{D}}^{2} \hat{f}_{I}-g^{2} f_{I J}{ }^{L} f_{K L}{ }^{M} \Phi^{J} \Phi^{K} \hat{f}_{M}-8 C_{I J K} \breve{\mathfrak{D}}^{2}\left(\Phi^{J} \Phi^{K} / H\right)=0 .
$$

\footnotetext{
${ }^{10}$ See also ref. [4].

${ }^{11}$ We have rescaled the 3 -dimensional fields by a factor of $-1 /(2 \sqrt{6})$ to conform to the normalization of the fields in $\mathcal{N}=2, d=4$ supergravity. See appendix A.

${ }^{12}$ See ref. [16] for the $\mathrm{SU}(2)$ case and ref. [25] and references therein for more general gauge groups.
} 
Defining

$$
\hat{f}_{I} \equiv L_{I}+8 C_{I J K} \Phi^{J} \Phi^{K} / H,
$$

and using the condition eq. (2.10) we find a linear equation for the functions $L_{I}$ :

$$
\breve{\mathfrak{D}}^{2} L_{I}-g^{2} f_{I J}^{L} f_{K L}^{M} \Phi^{J} \Phi^{K} L_{M}=0 .
$$

Finally, let us consider eq. (3.10). Defining $\hat{\omega}$ as

$$
\hat{\omega}=\omega_{5}(d z+\chi)+\omega, \quad \text { where } \quad \omega=\omega_{\underline{r}} d x^{r},
$$

eq. (3.10) gives an equation for $\omega_{5}$ whose general solution is

$$
\omega_{5}=M+16 \sqrt{2} H^{-2} C_{I J K} \Phi^{I} \Phi^{J} \Phi^{K}+3 \sqrt{2} H^{-1} L_{I} \Phi^{I}, \quad \text { where } \quad d \star_{3} d M=0,
$$

and the following equation for $\omega$ :

$$
\star_{3} d \omega=H d M-M d H+3 \sqrt{2}\left(\Phi^{I} \breve{\mathfrak{D}} L_{I}-L_{I} \breve{\mathfrak{D}} \Phi^{I}\right),
$$

whose integrability condition $d^{2} \omega=0$ is satisfied wherever the above equations for $H, M, \Phi^{I}, L_{I}$ are satisfied.

Summarizing: we have identified a set of $z$-independent functions $M, H, \Phi^{I}, L_{I}$ and 1 -forms $\omega, A^{I}, \chi$ in $\mathbb{E}^{3}$ in terms of which we can write all the building blocks of the 5 dimensional timelike supersymmetric solutions admitting an isometry as follows:

$$
\begin{aligned}
h_{I} / \hat{f} & =L_{I}+8 C_{I J K} \Phi^{J} \Phi^{K} / H, \\
\hat{\omega} & =\omega_{5}(d z+\chi)+\omega, \\
\omega_{5} & =M+16 \sqrt{2} H^{-2} C_{I J K} \Phi^{I} \Phi^{J} \Phi^{K}+3 \sqrt{2} H^{-1} L_{I} \Phi^{I}, \\
\hat{A}^{I} & =2 \sqrt{6}\left[H^{-1} \Phi^{I}(d z+\chi)-\breve{A}^{I}\right], \\
\hat{F}^{I} & =2 \sqrt{6} H^{-1}\left[\breve{\mathfrak{D}} \Phi^{I} \wedge(d z+\chi)-\star_{3} H \breve{\mathfrak{D}} \Phi^{I}\right],
\end{aligned}
$$

provided that they satisfy the following set of equations:

$$
\begin{aligned}
d \star_{3} d M & =0, \\
\star_{3} d H-d \chi & =0, \\
\star_{3} \breve{\mathfrak{D}} \Phi^{I}-\breve{F}^{I} & =0, \\
\breve{\mathfrak{D}}^{2} L_{I}-g^{2} f_{I J}{ }^{L} f_{K L}{ }^{M} \Phi^{J} \Phi^{K} L_{M} & =0, \\
\star_{3} d \omega-\left\{H d M-M d H+3 \sqrt{2}\left(\Phi^{I} \breve{\mathfrak{D}} L_{I}-L_{I} \breve{\mathfrak{D}} \Phi^{I}\right)\right\} & =0 .
\end{aligned}
$$

For symmetric real special manifolds we can use eq. (3.8) to write the metric function $\hat{f}$ explicitly in terms of the tensor $C_{I J K}$ and the functions $M, H, \Phi^{I}, L_{I}$ :

$$
\begin{aligned}
\hat{f}^{-3}= & 3^{3} C^{I J K} L_{I} L_{J} L_{K}+3^{4} \cdot 2^{3} C^{I J K} C_{K L M} L_{I} L_{J} \Phi^{L} \Phi^{M} / H \\
& +3 \cdot 2^{6} L_{I} \Phi^{I} C_{J K L} \Phi^{J} \Phi^{K} \Phi^{L} / H^{2}+2^{9}\left(C_{I J K} \Phi^{I} \Phi^{J} \Phi^{K}\right)^{2} / H^{3} .
\end{aligned}
$$


Let us compare the above formulae with those of the ungauged case (in ref. [19] in our conventions). It is easy to see that all the functions $M, H, \Phi^{I}, L_{I}$ become standard harmonic functions in $\mathbb{E}^{3}$. Furthermore, the functions $\Phi^{I}$ are related to the functions $K^{I}$ used in that reference by

$$
\Phi^{I}=+\frac{1}{2 \sqrt{2}} K^{I}
$$

\subsubsection{Dimensional reduction of the timelike supersymmetric solutions with one isometry}

The supersymmetric solutions that admit an additional isometry can be dimensionally reduced to supersymmetric solutions of $\mathcal{N}=2, d=4$ supergravity using the formulae in appendix A. ${ }^{13}$ Performing explicitly this reduction will allow us to simplify the tasks of oxidation and reduction of supersymmetric solutions.

First of all, the metric of the 4-dimensional solutions obtained through the dimensional reduction takes the conventional conformastationary form of the timelike supersymmetric solutions of the $\mathcal{N}=2, d=4$ theory

$$
d s^{2}=e^{2 U}(d t+\omega)^{2}-e^{-2 U} d x^{r} d x^{r},
$$

where the 1-form $\omega=\omega_{\underline{r}} d x^{r}$ is precisely the 1-form given in eq. (3.25) and the metric function $e^{-2 U}$ is given by

$$
e^{-2 U}=2 \sqrt{\frac{(\hat{f}-1 H)^{3}-\left(\omega_{5} H^{2}\right)^{2}}{4 H^{2}}} .
$$

We can compare the equations satisfied by the building blocks of the timelike supersymmetric solutions of gauged $\mathcal{N}=1, d=5$ supergravity (3.31)-(3.35) with the equations satisfied by the building blocks of the timelike supersymmetric solutions of gauged $\mathcal{N}=2, d=4$ supergravity ref. [3, 13], which we rewrite here for convenience adapting slightly the notation to avoid confusion with the different accents used to distinguish the different gauge fields:

$$
\begin{aligned}
-\frac{1}{\sqrt{2}} \star_{3} \breve{\mathfrak{D}} \mathcal{I}^{\Lambda}-\breve{F}^{\Lambda} & =0, \\
\breve{\mathfrak{D}}^{2} \mathcal{I}_{\Lambda}-\frac{1}{2} g^{2} f_{\Lambda \Sigma}{ }^{\Omega} f_{\Delta \Omega}{ }^{\Gamma} \mathcal{I}^{\Sigma} \mathcal{I}^{\Delta} \mathcal{I}_{\Gamma} & =0, \\
\star_{3} d \omega-2\left[\mathcal{I}_{\Lambda} \breve{\mathfrak{D}} \mathcal{I}^{\Lambda}-\mathcal{I}^{\Lambda} \breve{\mathfrak{D}} \mathcal{I}_{\Lambda}\right] & =0,
\end{aligned}
$$

where $\breve{\mathfrak{D}}$ is the gauge covariant derivative associated to the modified gauge connection in $\mathbb{E}^{3}$

$$
\breve{A}_{\underline{m}}^{\Lambda} \equiv A^{\Lambda}{ }_{\underline{m}}-\omega_{\underline{m}} A^{\Lambda}{ }_{t} .
$$

The notation that we are using has implicit the identification of the gauge potentials $\breve{A}$ coming from 5 and 4 dimensions, except for $\Lambda=0$. Using the formulae in appendix A

\footnotetext{
${ }^{13}$ These formulae are valid for any field configuration, supersymmetric or not.
} 
with the modifications explained in the last paragraph we can identify ${ }^{14}$

$$
\chi_{\underline{m}}=-2 \sqrt{2} \breve{A}^{0}{ }_{\underline{m}},
$$

which leads to the identifications

$$
\Phi^{I}=-\frac{1}{\sqrt{2}} \mathcal{I}^{I+1}, \quad L_{I}=\frac{2}{3} \mathcal{I}_{I+1}, \quad H=2 \mathcal{I}^{0}, \quad M=-\mathcal{I}_{0} .
$$

These are the only formulae we need to relate timelike supersymmetric solutions in $\mathcal{N}=$ $1, d=5$ supergravity with one additional isometry to timelike supersymmetric solutions in cubic model of $\mathcal{N}=2, d=4$ supergravity with $\mathcal{I}^{0} \neq 0 .{ }^{15}$

For symmetric real special scalar manifolds we can use the explicit form of $\hat{f}$ in eq. (3.36) together with the expression for $\omega_{5}$ in eq. (3.28) to get

$$
\begin{aligned}
e^{-2 U}=2 & \left\{\frac{3^{3}}{4} H C^{I J K} L_{I} L_{J} L_{K}-2^{7 / 2} M C_{I J K} \Phi^{I} \Phi^{J} \Phi^{K}+2 \cdot 3{ }^{4} C^{I J K} C_{K L M} L_{I} L_{J} \Phi^{L} \Phi^{M}\right. \\
& \left.-\frac{3^{2}}{2}\left(L_{I} \Phi^{I}\right)^{2}-\frac{3}{\sqrt{2}} H M L_{I} \Phi^{I}-\frac{1}{4} M^{2} H^{2}\right\}^{1 / 2}
\end{aligned}
$$

Then, using the identifications eqs. (3.45) together with the second of eqs. (A.1) we get

$$
\begin{aligned}
e^{-2 U}=2 & \left\{\left(d^{i j k} \mathcal{I}_{j} \mathcal{I}_{l}-\frac{2}{3} \mathcal{I}_{0} \mathcal{I}^{i}\right)\left(d_{i l m} \mathcal{I}^{l} \mathcal{I}^{m}+\frac{2}{3} \mathcal{I}^{0} \mathcal{I}_{i}\right)+\frac{4}{9} \mathcal{I}^{0} \mathcal{I}_{0} \mathcal{I}^{i} \mathcal{I}_{i}\right. \\
& \left.-\left(\mathcal{I}^{0} \mathcal{I}_{0}+\mathcal{I}^{i} \mathcal{I}_{i}\right)^{2}\right\}^{1 / 2} .
\end{aligned}
$$

\subsection{Null supersymmetric solutions}

The general form of the null supersymmetric solutions of $\mathcal{N}=1, d=5$ SEYM is quite involved [1], but it simplifies dramatically when one assumes the existence of an additional isometry so that all the fields are independent of the two null coordinates $u$ and $v$. These are the solutions which will become timelike supersymmetric solutions of $\mathcal{N}=2, d=4$ SEYM upon dimensional reduction and, therefore, we are going to describe only these.

\subsection{1 $u$-independent null supersymmetric solutions}

The metric of the general null supersymmetric solutions of $\mathcal{N}=1, d=5$ SEYM can always brought into the form $[1]^{16}$

$$
d s^{2}=2 \ell d u(d v+K d u+\sqrt{2} \omega)-\ell^{-2} d x^{r} d x^{r},
$$

where the functions $\ell, K$ and the 1 -form $\omega=\omega_{\underline{r}} d x^{r}$ are $v$-independent. We are going to assume also $u$-independence of all the fields throughout.

\footnotetext{
${ }^{14}$ The 0th components are never gauged if the dimensional reduction is simple (not generalized).

${ }^{15}$ Those with $\mathcal{I}^{0}=0$ are related to null supersymmetric 5-dimensional solutions.

${ }^{16}$ We have changed the notation and normalization with respect to [1] to avoid possible confusions between the objects that appear in the null and timelike cases.
} 
After the partial gauge fixing $A^{I} \underline{v}=0$, the gauge fields are decomposed as ${ }^{17}$

$$
A^{I}=A_{\underline{u}}^{I} d u-2 \sqrt{6} \breve{A}^{I}, \quad \breve{A}^{I}=\breve{A}_{\underline{r}}^{I} d x^{r},
$$

and the vector field strengths take the form ${ }^{18}$

$$
F^{I}=\left(\sqrt{2 / 3} \ell^{2} h^{I} \star_{3} d \omega-\psi^{I}\right) \wedge d u+\sqrt{3} \star_{3} \breve{\mathfrak{D}}\left(h^{I} / \ell\right),
$$

where the $\psi^{I}$ are some 1-forms in $\mathbb{E}^{3}$ satisfying

$$
h_{I} \psi^{I}=0,
$$

to be determined and $\breve{\mathfrak{D}}$ is the gauge-covariant derivative on $\mathbb{E}^{3}$ with respect to the connection $\breve{A}^{I}$.

Finally, the scalar fields will be determined by the equations obeyed by the scalar functions $h^{I}$, which follow from the equations of motion. ${ }^{19}$

Let us start by analyzing the Bianchi identities of the vector field strength. They lead to the following two sets of equations:

$$
\begin{aligned}
-\frac{1}{2 \sqrt{2}} \star_{3} \breve{\mathfrak{D}}\left(h^{I} / \ell\right)-\breve{F}^{I} & =0, \\
\breve{\mathfrak{D}} A_{\underline{u}}^{I}-\sqrt{2 / 3} \ell^{2} h^{I} \star_{3} d \omega+\psi^{I} & =0 .
\end{aligned}
$$

Eq. (3.52) is the Bogomol'nyi equation on $\mathbb{E}^{3}$ and, thus, we define the Higgs field

$$
\Sigma^{I} \equiv-\frac{1}{2 \sqrt{2}} h^{I} / \ell
$$

Multiplying eq. (3.53) by $h_{I}$ and using eq. (3.51) together with $h_{I} h^{I}=1$ we get the equation that defines $\omega$

Defining the functions

$$
d \omega=\sqrt{3 / 2} \ell^{-2} \star_{3}\left\{h_{I} \breve{\mathfrak{D}} A^{I}{ }_{\underline{u}}\right\} .
$$

$$
K_{I} \equiv C_{I J K} \Sigma^{J} A^{K} \underline{u},
$$

the above equation takes a much more familiar form

$$
d \omega=4 \sqrt{6} \star_{3}\left\{\Sigma^{I} \breve{\mathfrak{D}} K_{I}-K_{I} \breve{\mathfrak{D}} \Sigma^{I}\right\},
$$

whose integrability condition is

$$
\Sigma^{I} \breve{\mathfrak{D}}^{2} K_{I}=0 .
$$

Given the functions $\Sigma^{I}, K_{I}$ and the gauge fields $\breve{A}^{I}$ we can solve this equation for $\omega$. It should be possible to find the functions $A^{I} \underline{u}$ in terms of $\Sigma^{I}, K_{I}{ }^{20}$ and, plugging these result in eq. (3.53), compute directly the 1 -forms $\psi^{I}$.

\footnotetext{
${ }^{17}$ As the notation suggests, the gauge fields $\breve{A}^{I}$ are the same as the $\mathcal{N}=2, d=4$ fields denoted with the same symbols, according to the general formulae of appendix A. The same is true of the 1-form $\omega$.

${ }^{18}$ All the operators in the r.h.s. are defined in $\mathbb{E}^{3}$.

${ }^{19}$ The field configurations that we have just described are automatically supersymmetric, but not necessarily solutions of all the equations of motion and Bianchi identities [1].

${ }^{20}$ This will certainly be the case for the particular model we are going to study, but we have not found (even for just the symmetric case) a general way of solving eq. (3.56) for $A^{I} \underline{u}$.
} 
From the Maxwell equations one obtains the equations that determine the functions $K_{I}$ :

$$
\breve{\mathfrak{D}}^{2} K_{I}-g^{2} f_{I J}{ }^{L} f_{K L}{ }^{M} \Sigma^{J} \Sigma^{K} K_{M}=0,
$$

from which the integrability condition eq. (3.58) follows automatically.

Finally, defining

$$
N \equiv K-\sqrt{2} A^{I} \underline{u} K_{I},
$$

the last non-trivial equation of motion, from the Einstein equations, takes the simple form

$$
\nabla^{2} N=0 .
$$

Summarizing: we have identified a set of $u$-independent functions $\Sigma^{I}, K_{I}, N$ and 1-forms $\omega, \breve{A}^{I}$ on $\mathbb{E}^{3}$ in terms of which we can write all the building blocks of the 5 -dimensional $u$-independent null supersymmetric solutions, assuming we can solve eq. (3.56) for $A^{I} \underline{u}$, as follows:

$$
\begin{aligned}
h^{I} / \ell & =-2 \sqrt{2} \Sigma^{I}, \\
K & =N+\sqrt{2} A_{\underline{u}}^{I} K_{I}, \\
A^{I} & =A^{I}{ }_{\underline{u}} d u+2 \sqrt{6} \breve{A}^{I}, \\
F^{I} & =\breve{\mathfrak{D}} A^{I}{ }_{\underline{u}} \wedge d u+\sqrt{3} \star_{3} \breve{\mathfrak{D}}\left(h^{I} / \ell\right),
\end{aligned}
$$

provided the following equations are satisfied: ${ }^{21}$

$$
\begin{aligned}
\star_{3} \breve{\mathfrak{D}} \Sigma^{I}-\breve{F}^{I} & =0, \\
\breve{\mathfrak{D}}^{2} K_{I}-g^{2} f_{I J}{ }^{L} f_{K L}{ }^{M} \Sigma^{J} \Sigma^{K} K_{M} & =0, \\
d \omega-4 \sqrt{6} \star_{3}\left\{\Sigma^{I} \breve{\mathfrak{D}} K_{I}-K_{I} \breve{\mathfrak{D}} \Sigma^{I}\right\} & =0, \\
\nabla^{2} N & =0 .
\end{aligned}
$$

Using eq. (2.1), we find a general expression for $\ell$ :

$$
\ell^{-3}=-2^{9 / 2} C_{I J K} \Sigma^{I} \Sigma^{J} \Sigma^{K} .
$$

\subsubsection{Dimensional reduction of the $u$-independent null supersymmetric solu- tions}

Using the general formulae in appendix A, the $u$-independent solutions that we have considered can be dimensionally reduced to timelike supersymmetric solutions of $\mathcal{N}=2, d=4$ SEYM along the spacelike coordinate $z$ defined by

$$
u=\frac{1}{\sqrt{2}}(t+z), \quad v=\frac{1}{\sqrt{2}}(t-z),
$$

with metrics of the form eq. (3.38) where the 1-form $\omega=\omega_{\underline{r}} d x^{r}$ is precisely the 1-form given in eq. (3.48) and the metric function $e^{-2 U}$ is given by

$$
e^{-2 U}=\sqrt{\ell^{-3}(1-K)}=\sqrt{-2^{9 / 2} C_{I J K} \Sigma^{I} \Sigma^{J} \Sigma^{K}\left(1-N-\sqrt{2} A^{I} \underline{u} K_{I}\right)} .
$$

\footnotetext{
${ }^{21}$ The gauge coupling constant is the 4 -dimensional one.
} 
In order to express entirely the metric function in terms of the functions $K_{I}, \Sigma^{I}, N$ we need to solve eq. (3.56) for $A_{\underline{p}}^{I}$ as a function of $K_{I}, \Sigma^{I}$, which we do not know how to do in general. We can still compare the equations satisfied by these functions (3.66)-(3.69) with those satisfied by $\mathcal{I}^{\Lambda}, \mathcal{I}_{\Lambda}$ in $\mathcal{N}=2, d=4$ SEYM (3.40)-(3.42) knowing that the vector fields $\breve{A}^{I}$ and the 1 -form $\omega$ are the same objects. We find that

$$
\Sigma^{I}=-\frac{1}{\sqrt{2}} \mathcal{I}^{I+1}, \quad K_{I}=-\frac{1}{2 \sqrt{3}} \mathcal{I}_{I+1}
$$

while $N$ must be proportional to either $\mathcal{I}^{0}$ or $\mathcal{I}_{0}$. Since a wave moving in the internal $z$ direction should give rise to a 4-dimensional electric charge, it must be

$$
N \sim \mathcal{I}_{0}
$$

but the precise coefficient cannot be determined from this comparison alone. We have to find a more explicit expression for $e^{-2 U}$.

\section{5-dimensional supersymmetric non-Abelian solutions of the SU(2)- gauged ST[2,5] model}

In this section we are going to consider a particular model of $\mathcal{N}=1, d=5$ supergravity that admits an $\mathrm{SU}(2)$ gauging. This model is related to the $\mathrm{SU}(2)$-gauged $\mathrm{ST}[2,5]$ model of $\mathcal{N}=2, d=4$ supergravity some of whose solutions we have studied in ref. [12]. We will use the relations derived in the previous section to find relations between the non-Abelian supersymmetric solutions of both theories.

We start by describing the 4- and 5-dimensional models and their $\mathrm{SU}(2)$ gauging.

\subsection{The models}

The ST $[2,5]$ model is a cubic model of $\mathcal{N}=2, d=4$ supergravity coupled to 5 vector multiplets i.e. a model with a prepotential of the form

$$
\mathcal{F}=-\frac{1}{3 !} \frac{d_{i j k} \mathcal{X}^{i} \mathcal{X}^{j} \mathcal{X}^{k}}{\mathcal{X}^{0}}, \quad i=1,2 \cdots, 5
$$

where the fully symmetric tensor $d_{i j k}$ has as only non-vanishing components

$$
d_{1 \alpha \beta}=\eta_{\alpha \beta}, \quad \text { where } \quad\left(\eta_{\alpha \beta}\right)=\operatorname{diag}(+-\cdots-), \quad \text { and } \alpha, \beta=2, \cdots, 5 .
$$

The 5 complex scalars parametrize the coset space

$$
\frac{\mathrm{SL}(2, \mathbb{R})}{\mathrm{SO}(2)} \times \frac{\mathrm{SO}(2,4)}{\mathrm{SO}(2) \times \mathrm{SO}(4)}
$$

and the group $\mathrm{SO}(3)$ acts in the adjoint on the coordinates $\alpha=3,4,5$. These are the directions we are going to gauge and we will denote them with capital $A, B, \ldots$. This is the only information we need in order to construct supersymmetric solutions, but more details 
on the construction of this theory can be found in ref. [12]. We will need the form of the metric function in terms of the functions $\mathcal{I}^{M}$ :

$$
e^{-2 U}=2 \sqrt{\left(\mathcal{I}^{\alpha} \mathcal{I}^{\beta} \eta_{\alpha \beta}+2 \mathcal{I}^{0} \mathcal{I}_{1}\right)\left(\mathcal{I}_{\alpha} \mathcal{I}_{\beta} \eta^{\alpha \beta}-2 \mathcal{I}^{1} \mathcal{I}_{0}\right)-\left(\mathcal{I}^{0} \mathcal{I}_{0}-\mathcal{I}^{1} \mathcal{I}_{1}+\mathcal{I}^{\alpha} \mathcal{I}_{\alpha}\right)^{2}}
$$

The models of the ST $[2, n]$ family are related to the effective theory of the Heterotic string and compactified on $T^{6}$ by a consistent truncation: the 10-dimensional effective theory is $\mathcal{N}=1, d=10$ supergravity coupled to 16 10-dimensional vector multiplets with gauge group $\mathrm{U}(1)$. Upon dimensional reduction on a generic $T^{6}$ one gets $\mathcal{N}=4, d=4$ supergravity coupled to $16+6=22$ vector multiplets, whose duality group is

$$
\frac{\mathrm{SL}(2, \mathbb{R})}{\mathrm{SO}(2)} \times \frac{\mathrm{SO}(6,22)}{\mathrm{SO}(6) \times \mathrm{SO}(22)} .
$$

Observe that $\mathrm{SO}(6)$ acts on the 6 vectors in the supergravity multiplet and $\mathrm{SO}(22)$ on the 22 matter vector fields. The coset $\mathrm{SL}(2, \mathbb{R}) / \mathrm{SO}(2)$ is parametrized by the only scalar in the supergravity multiplet. A consistent truncation to $\mathcal{N}=2, d=4$ eliminates 4 vectors from the $\mathcal{N}=4$ supergravity multiplet and one of the remaining two vectors becomes a matter vector field from the $\mathcal{N}=2$ point of view and comes in the same multiplet as the complex scalar that parametrizes the coset space $\mathrm{SL}(2, \mathbb{R}) / \mathrm{SO}(2)$. The result is a $\mathrm{ST}[2,23]$ model from which one can consistently eliminate vector multiplets to arrive to the $\mathrm{ST}[2,5]$ model we are dealing with.

This is the story at a generic point in the moduli space of the Heterotic strings on $T^{6}$. At certain points, though, there is an enhancement of gauge symmetry usually associated to an increase in the number of massless vector fields that we must take into account in the effective theory. Our SU(2)-gauged model of $\mathcal{N}=2, d=4$ supergravity can be interpreted as the effective theory describing the simplest of these situations in which the enhancement of gauge symmetry arises in the sector of the 16 original 10-dimensional vector fields.

The ST $[2,5]$ model is related to a model of $\mathcal{N}=1, d=5$ supergravity coupled to 4 vector multiplets determined by the tensor $C_{i-1, j-1, k-1}=\frac{1}{6} d_{i j k}$ so its only non-vanishing components are

$$
C_{0 x y}=\frac{1}{6} \eta_{x y}, \text { where }\left(\eta_{x y}\right)=\operatorname{diag}(+-\cdots-), \quad \text { and } \quad x, y=1, \cdots, 4 .
$$

The 4 real scalars in the vector multiplets parametrize the coset space

$$
\frac{\mathrm{SO}(1,3)}{\mathrm{SO}(3)} \text {. }
$$

Now the group $\mathrm{SO}(3)$ acts in the adjoint on the coordinates $x=2,3,4$ and, if we gauge it, the theory goes to the gauged 4-dimensional model we just discussed. It should be obvious after the 4-dimensional discussion that this model can be interpreted as a truncation of the effective theory of the Heterotic string compactified on $T^{5}$.

Again, we do not need many more details of the theory in order to construct supersymmetric solutions. For timelike supersymmetric solutions admitting an additional isometry we will need the metric function, which follows directly from the generic expression 
eq. (3.36)

$$
\begin{aligned}
\hat{f}^{-1}=H^{-1} & \left\{\frac { 1 } { 4 } ( 6 H L _ { 0 } + 8 \eta _ { x y } \Phi ^ { x } \Phi ^ { y } ) \left[9 H^{2} \eta^{x y} L_{x} L_{y}+48 H \Phi^{0} L_{x} \Phi^{x}\right.\right. \\
& \left.\left.+64\left(\Phi^{0}\right)^{2} \eta_{x y} \Phi^{x} \Phi^{y}\right]\right\}^{1 / 3}
\end{aligned}
$$

This metric function and the 4-dimensional one $e^{-2 U}$ are related by eq. (3.39) using eq. (3.28) and the relations between the functions $\mathcal{I}^{M}$ and $H, M, L_{I}, \Phi^{I}$ in eqs. (3.45), which we rewrite for this specific pair of models for convenience:

$$
\begin{aligned}
& H=2 \mathcal{I}^{0}, \quad \Phi^{0}=-\frac{1}{\sqrt{2}} \mathcal{I}^{1}, \quad \Phi^{1}=-\frac{1}{\sqrt{2}} \mathcal{I}^{2}, \quad \Phi^{A}=-\frac{1}{\sqrt{2}} \mathcal{I}^{A}, \\
& M=-\mathcal{I}_{0}, \quad L_{0}=\frac{2}{3} \mathcal{I}_{1}, \quad L_{1}=\frac{2}{3} \mathcal{I}_{2}, \quad L_{A}=\frac{2}{3} \mathcal{I}_{A},
\end{aligned}
$$

For $u$-independent null supersymmetric solutions we first need to solve eq. (3.56) for $A^{I} \underline{u}$. For this model, we find

$$
A_{\underline{u}}^{0}=6 \frac{\Sigma^{x} K_{x}-\Sigma^{0} K_{0}}{(\eta \Sigma \Sigma)}, \quad A_{\underline{u}}^{x}=6 \frac{\eta^{x y} K_{y}(\eta \Sigma \Sigma)-\Sigma^{x}\left(\Sigma^{y} K_{y}-\Sigma^{0} K_{0}\right)}{\Sigma^{0}(\eta \Sigma \Sigma)},
$$

where $(\eta \Sigma \Sigma) \equiv \eta_{x y} \Sigma^{x} \Sigma^{y}$, so that

$$
e^{-2 U}=2 \sqrt{\left(\mathcal{I}^{\alpha} \mathcal{I}^{\beta} \eta_{\alpha \beta}\right)\left[\mathcal{I}_{\alpha} \mathcal{I}_{\beta} \eta^{\alpha \beta}+\mathcal{I}^{1}(1-N)\right]-\left(-\mathcal{I}^{1} \mathcal{I}_{1}+\mathcal{I}^{\alpha} \mathcal{I}_{\alpha}\right)^{2}},
$$

and we arrive at the following identifications

$$
\begin{aligned}
& 0=\mathcal{I}^{0}, \quad \Sigma^{0}=-\frac{1}{\sqrt{2}} \mathcal{I}^{1}, \quad \Sigma^{1}=-\frac{1}{\sqrt{2}} \mathcal{I}^{2}, \quad \Sigma^{A}=-\frac{1}{\sqrt{2}} \mathcal{I}^{A}, \\
& N=1+2 \mathcal{I}_{0}, \quad K_{0}=-\frac{1}{2 \sqrt{3}} \mathcal{I}_{1}, \quad K_{1}=-\frac{1}{2 \sqrt{3}} \mathcal{I}_{2}, \quad K_{A}=-\frac{1}{2 \sqrt{3}} \mathcal{I}_{A} \text {. }
\end{aligned}
$$

\subsection{The solutions}

We are ready to put to work the machinery developed in the previous sections. We are going to consider the simplest cases first.

\subsubsection{A simple $5 d$ black hole with non-Abelian hair}

In order to add non-Abelian fields to our solutions it is exceedingly useful to consider metrics with one additional isometry, because, then, we can make use of our knowledge of the spherically symmetric solutions of the Bogomol'nyi equations of the SU(2) YMH system found by Protogenov in ref. [16]. However, this isometry cannot be translational if we want to find spherically-symmetric black holes because, then, the full 5-dimensional solution will have a translational isometry. Thus, we will start with the choice $H=1 / r$ $\left(r^{2}=y^{r} y^{r}\right)^{22}$ which, as we have shown in ref. [4], relates the colored monopole solution ${ }^{23}$ to the the BPST instanton, which is spherically symmetric in $\mathbb{E}^{4}$.

\footnotetext{
${ }^{22}$ We need to distinguish between the Cartesian coordinates in $\mathbb{E}^{3}$, which we will denote by $y^{r}$ and the Cartesian coordinates in $\mathbb{E}^{4}$, which we will denote by $x^{m}$. The former are not a simple subset of the latter.

${ }^{23}$ This monopole is characterized by a vanishing magnetic charge.
} 
We are, thus, going to consider a configuration with the following non-vanishing functions:

$$
H=\frac{1}{r}, \quad L_{0}=A_{0}+\frac{q_{0}}{4 r}, \quad L_{1}=A_{1}+\frac{q_{1}}{4 r}, \quad \Phi^{A}=-f(r) \delta^{A}{ }_{r} y^{r},
$$

where $q_{0}, q_{1}$ are electric charges in some convenient normalization, $A_{0}, A_{1}$ are constants to be determined through the normalization of the metric and the scalar fields at infinity and $f(r)$ is the function (not to be mistaken by $\hat{f}$ ) that characterizes the Higgs field in the spherically-symmetric monopole solutions of ref. $\left.[16]^{24}\right)$.

The next step consists in finding the 1-forms $\chi, \breve{A}^{I}, \omega$ and functions $L_{I}$ that satisfy eqs. (3.32)-(3.35) for the above non-vanishing functions. $\omega$ is closed and can be set to zero, the functions $L_{I}$ can also be set to zero while ${ }^{25}$

$$
\chi=\cos \theta d \psi, \quad \breve{A}^{A}=h(r) \varepsilon^{A}{ }_{r s} y^{r} d y^{s},
$$

where $h(r)$ is the function that characterizes the gauge field of the monopole solution (see appendix B)). The spacetime metric is, then,

$$
d s^{2}=\hat{f}^{2} d t^{2}-\hat{f}^{-1}\left[r(d \varphi+\cos \theta d \psi)^{2}+\frac{1}{r}\left(d r^{2}+r^{2} d \Omega_{(2)}^{2}\right)\right],
$$

where

$$
d \Omega_{(2)}^{2}=d \theta^{2}+\sin ^{2} \theta d \psi^{2},
$$

and, upon the change of coordinates $r=\rho^{2} / 4$, it becomes

$$
d s^{2}=\hat{f}^{2} d t^{2}-\hat{f}^{-1} d x^{m} d x^{m}, \text { where } d x^{m} d x^{m}=d \rho^{2}+\rho^{2} d \Omega_{(3)}^{2} .
$$

For this configuration, the metric function eq. (4.8) is given by

$$
\hat{f}^{-1}=3 \sqrt[3]{\frac{1}{2}\left(L_{0}-\frac{4}{3} r^{3} f^{2}\right)\left(L_{1}\right)^{2}},
$$

and it immediately follows that in order for the solution to be asymptotically regular, the monopole must be the colored one for which $r^{3} f_{\lambda}^{2} \sim 1 / r$, because for all the rest $r^{3} f^{2} \sim r$ (see appendix B). With this choice, ${ }^{26}$ as shown in ref. [4], ${ }^{27}$ the gauge field $\hat{A}^{A}=\hat{A}^{A}{ }_{\underline{m}} d x^{m}$ that follows from the use of eq. (3.29) is that of a BPST instanton in $\mathbb{E}^{4}$ :

$$
\hat{A}^{A}=\frac{1}{\tilde{g}} \frac{1}{1+\lambda^{2} \rho^{2} / 4} v_{L}^{A},
$$

where $v_{L}^{A}$ are the $\mathrm{SU}(2)$ left-invariant Maurer-Cartan 1 -forms. ${ }^{28}$ Since the scalar functions $h^{A}$ vanish for this configuration, the full 5-dimensional vector fields are, according to

\footnotetext{
${ }^{24}$ See appendix B in which we have written all of Protogenov's solutions.

${ }^{25}$ The choice of angular coordinates is conditioned by the relation between the monopole and instanton as explained in ref. [4]. We will identify the compact coordinate $z$ with the angular coordinate $\varphi$.

${ }^{26}$ We are going to study the consequences of the other choices in section 4.2.3.

${ }^{27}$ More specifically, the gauge field one gets is $\hat{A}_{L}^{A(+)}$.

${ }^{28}$ In our conventions, these are given by

$$
\left\{\begin{array}{l}
v_{L}^{1}=\sin \psi d \theta-\sin \theta \cos \psi d \varphi, \\
v_{L}^{2}=-\cos \psi d \theta-\sin \theta \sin \psi d \varphi, \quad \text { and } \quad d v_{L}^{A}+\frac{1}{2} \epsilon_{A B C} v_{L}^{B} \wedge v_{L}^{C}=0 \\
v_{L}^{3}=-(d \psi+\cos \theta d \varphi),
\end{array}\right.
$$


eq. (3.12), given by

$$
\begin{aligned}
A^{0} & =\frac{3^{5 / 2}}{2}\left(L_{1}\right)^{2} \hat{f}^{3} d t \\
A^{1} & =3^{5 / 2} L_{1}\left(L_{0}-\frac{4}{3} r^{3} f_{\lambda}^{2}\right) \hat{f}^{3} d t, \\
A^{A} & =\frac{1}{\tilde{g}} \frac{1}{1+\lambda^{2} \rho^{2} / 4} v_{L}^{A} .
\end{aligned}
$$

Finally, the only non-vanishing scalar is given by by

$$
\phi \equiv h_{1} / h_{0}=\frac{L_{1}}{L_{0}-\frac{4}{3} r^{3} f_{\lambda}^{2}} .
$$

The integration constants are readily identified in terms of the asymptotic value of the scalar as

$$
A_{0}=\frac{2^{1 / 3}}{3} \phi_{\infty}^{-2 / 3}, \quad A_{1}=\frac{2^{1 / 3}}{3} \phi_{\infty}^{1 / 3}
$$

while the mass and the area of the event horizon are given by

$$
\begin{aligned}
M & =2^{-1 / 3} 3^{1 / 2}\left[\phi_{\infty}^{2 / 3} q_{0}+2 \phi_{\infty}^{-1 / 3} q_{1}\right], \\
\frac{A}{2 \pi^{2}} & =\sqrt{\frac{3^{3}}{2}\left(q_{0}-\frac{2^{7}}{\tilde{g}^{2}}\right)\left(q_{1}\right)^{2}} .
\end{aligned}
$$

This solution can be understood as the result of the addition of a BPST instanton to a standard 2-charge Abelian solution. This addition does not produce any observable effects at spatial infinity, like, for instance, a change in the mass, but does produce a change in the near-horizon geometry and in the entropy.

The metric function of the 4-dimensional solution $e^{-2 U}$ that one obtains by dimensional reduction is related to the metric function of the 5 -dimensional solution by

$$
e^{-4 U}=\frac{1}{r} \hat{f}^{-3}
$$

which implies that the 4- and 5-dimensional solutions cannot be asymptotically flat at the same time. In particular, with the choice made above (corresponding to a colored monopole in $d=4) e^{-2 u} \sim r^{-1 / 2}$ at spatial infinity, a behavior that does not correspond to any known vacuum. With the monopoles we discarded, however, we get an asymptoticallyflat solution. The near-horizon behavior is simultaneously good in $d=4$ and $d=5$.

\subsubsection{A rotating $5 d$ black hole with non-Abelian hair}

In the context of timelike supersymmetric solutions of $\mathcal{N}=1, d=5$ supergravity rotation can be added by switching on the harmonic function $M$ [26]. More specifically, we add to the static solution we just constructed the harmonic function

$$
M=\frac{J / 2}{4 r},
$$


which only appears in eq. (3.28). The metric of the new solution is

$$
d s^{2}=\hat{f}^{2}\left[d t+\frac{J / 2}{4 r}(d \varphi+\cos \theta d \psi)\right]^{2}-\hat{f}^{-1}\left[r(d \varphi+\cos \theta d \psi)^{2}+\frac{1}{r}\left(d r^{2}+r^{2} d \Omega_{(2)}^{2}\right)\right],
$$

where the metric function $\hat{f}$ is still given by eq. (4.18). The scalar field $\phi$ and the nonAbelian vector field $A^{A}$ take the same value as in the static solution while the two Abelian vector fields are modified by the change

$$
d t \longrightarrow d t+\frac{J / 2}{4 r}(d \varphi+\cos \theta d \psi)
$$

which describes the presence of a magnetic dipole moment associated to the rotation.

Asymptotically, the only novelty is the off-diagonal term $\sim J / \rho^{2} d t(d \varphi+\cos \theta d \psi)$ which corresponds to identical values of the two Casimirs of the angular momentum, both proportional to $J$, so this solution is a non-Abelian generalization of the Breckenridge-MyersPeet-Vafa (BMPV) spinning black hole $[27,28]$. The mass has the same expression in terms of the charges as in the static case.

In the near-horizon limit, if the behavior of the metric function $\hat{f}$ is

$$
\hat{f}^{-1} \sim R^{2} / r
$$

for some constant $R$, the metric can be rewritten in the form

$$
d s^{2} \sim R^{2} d \Pi_{(2)}^{2}-R^{2} d \Omega_{(2)}^{2}-R^{2}\left[\cos \alpha(d \varphi+\cos \theta d \psi)-\sin \alpha \frac{r}{R^{2}} d \phi\right]^{2},
$$

where $\phi$ is the rescaled time coordinate, defined as follows

$$
\phi \equiv t / X, \quad X / R \equiv \sqrt{1-\left[J /(2 R)^{3}\right]^{2}} \equiv \cos \alpha, \quad(2 R)^{3} \equiv \sqrt{\frac{3^{3}}{2}\left(q_{0}-\frac{2^{7}}{\tilde{g}^{2}}\right)\left(q_{1}\right)^{2}},
$$

and $d \Pi_{(2)}^{2}, d \Omega_{(2)}^{2}$ are the metrics of the 2-dimensional Anti-de Sitter and sphere of unit radius

$$
d \Pi_{(2)}^{2} \equiv\left(\frac{r}{R^{2}}\right)^{2} d \phi^{2}-\frac{d r^{2}}{r^{2}} .
$$

The constant-time sections of the event horizon are squashed 3 -spheres with metric

$$
-d s^{2}=R^{2}\left\{\cos ^{2} \alpha(d \varphi+\cos \theta d \psi)^{2}+d \Omega_{(2)}^{2}\right\}
$$

and area

$$
\frac{A}{2 \pi^{2}}=\sqrt{\frac{3^{3}}{2}\left(q_{0}-\frac{2^{7}}{\tilde{g}^{2}}\right)\left(q_{1}\right)^{2}-J^{2}} .
$$

\subsubsection{A more general solution}

In section 4.2.1 we used the colored monopole solution in order to obtain an asymptotically flat black-hole solution in the simplest way. However, we can also use the monopoles in the 2-parameter family, for which, asymptotically, $r^{3} f^{2} \sim r$ if we switch on additional 
harmonic functions and choose the values of the integration constants appropriately so that the metric functions $\hat{f}(r), \omega_{5}, \omega$ give an asymptotically-flat solution.

Throughout the following discussion, it is convenient to have the explicit form of these functions for $H=1 / r, \Phi^{A}=-f(r) \delta^{A}{ }_{r} y^{r}$ and $L_{A}=0$ at hand:

$$
\begin{aligned}
\hat{f}^{-3} & =27\left[\frac{1}{2} L_{0}+\frac{2}{3} r\left[\left(\Phi^{1}\right)^{2}-r^{2} f^{2}\right]\right]\left[\left(L_{1}\right)^{2}+\frac{16}{3} r \Phi^{0} L_{1} \Phi^{1}+\frac{64}{9}\left(r \Phi^{0}\right)^{2}\left[\left(\Phi^{1}\right)^{2}-r^{2} f^{2}\right]\right] \\
\omega_{5} & =M+8 \sqrt{2} r^{2} \Phi^{0}\left[\left(\Phi^{1}\right)^{2}-r^{2} f^{2}\right]+3 \sqrt{2} r L_{i} \Phi^{i} \\
\star_{3} d \omega & =\frac{1}{r} d M-M d \frac{1}{r}+3 \sqrt{2}\left(\Phi^{i} d L_{i}-L_{i} d \Phi^{i}\right),
\end{aligned}
$$

where $i=0,1$. Apart from the functions $H$ and $\Phi^{A}$, we are going to consider the following non-vanishing harmonic functions

$$
\left\{\Phi^{0}, \Phi^{1}, L_{0}, L_{1}, M\right\}
$$

with

$$
\Phi^{0,1}=A^{0,1}+\frac{p^{0,1}}{4 r}, \quad L_{0,1}=A_{0,1}+\frac{q_{0,1}}{4 r}, \quad M=a+\frac{b}{4 r}
$$

$\hat{f}^{-3}$ is a product of two factors. Our strategy will be to make the constant piece of $\Phi^{1}$, $A^{1}$, cancel the constant piece in $r f(r), \mu / g$ so that $\left[\left(\Phi^{1}\right)^{2}-r^{2} f^{2}\right]$ is asymptotically $\mathcal{O}(1 / r):^{29}$

$$
A^{1}=\mu / g
$$

This ensures that the second term in $\hat{f}^{-3}$ diverges asymptotically at most as $\mathcal{O}(r)$ while the first is asymptotically constant. This constant can be made to vanish by choosing the constant piece of $L_{0}, A_{0}$, to be

$$
A_{0}=-\frac{8}{3} \frac{\mu}{g}\left(\frac{1}{g}+\frac{p^{1}}{4}\right)
$$

and now the first term is asymptotically $\mathcal{O}(1 / r)$ and $\hat{f}^{-3}$ is asymptotically constant.

Next, we require that all the $\mathcal{O}\left(r^{2}\right), \mathcal{O}(r)$ and $\mathcal{O}(1)$ terms in $\omega_{5}$ vanish. ${ }^{30}$ This gives two new relations ${ }^{31}$ between the constants $A_{i}, A^{i}$ and $a$. The vanishing of $\omega$ gives another relation between the same constants. Thus, requiring asymptotic flatness fixes the values of all these constants in terms of the Abelian charges $p^{i}, q_{i}$ and $\mu$ and $g$. Finally the normalization of the metric at infinity also fixes the value of $\mu$ and the solution has no free moduli!

\footnotetext{
${ }^{29}$ We choose the positive sign for simplicity.

${ }^{30}$ Observe that this does not imply the complete vanishing of $\omega_{5}$ : there are $\mathcal{O}(1 / r)$ terms that give angular momentum (which could be cancelled by the integration constant $b$ in $M)$ and also $\mathcal{O}\left(e^{-4 \mu r}\right)$ terms that cannot be cancelled. Therefore, the metric is not static even if the angular momentum is set to zero.

${ }^{31}$ The above values of $A_{0}$ and $A^{1}$ make the $\mathcal{O}\left(r^{2}\right)$ term vanish.
} 
The values of the integration constants $A_{0}, A^{1}$ has been given above and the values of the rest $\operatorname{are}^{32}$

$$
\begin{aligned}
A_{1} & =-\frac{88}{3} A^{0}\left(\frac{1}{g}+\frac{p^{1}}{4}\right) \\
A^{0} & =\left\{\frac{\left(16 p^{0}+4 g p^{0} p^{1}+g q^{1}\right)\left(4+g p^{1}\right)^{-1}}{40\left(3 q_{0}+\left(p^{1}\right)^{2}-\frac{16}{g^{2}}\right)\left(q_{0}+2\left(p^{1}\right)^{2}-\frac{32}{g^{2}}\right)}\right\}^{1 / 3}, \\
\mu & =A^{0}\left[\frac{32-2 g^{2}\left(p^{1}\right)^{2}-g^{2} q_{0}}{16 p^{0}+4 g p^{0} p^{1}+g q_{1}}\right], \\
a & =\sqrt{2} A^{0}\left[\frac{48}{g^{2}}+\frac{22 p^{1}}{g}+\frac{5\left(p^{1}\right)^{2}}{2}-\frac{3 q_{0}}{4}\right]-\sqrt{2}\left[\frac{22 \mu p^{0}}{g^{2}}+\frac{11 \mu p^{0} p^{1}}{2 g}+\frac{3 \mu q_{1}}{4 g}\right], \\
b & =J / 2-6 \sqrt{2}\left[\frac{p^{0}\left(p^{1}\right)^{2}}{2}+\frac{p^{0} q_{0}+p^{1} q^{1}}{8}-8 \frac{p^{0}}{g^{2}}\right]
\end{aligned}
$$

where $J$ is the angular momentum.

The mass of this solution is given by

$$
M=\frac{\pi A^{0}}{2 G}\left[3 q_{0}+\left(p^{1}\right)^{2}-\frac{16}{g^{2}}\right]\left[3 \frac{\mu}{g} q_{1}+8\left(\frac{1}{g}+\frac{p^{1}}{4}\right)\left(10 A^{0}\left(\frac{24}{g}+5 p^{1}\right)-9 \frac{\mu}{g} p^{0}\right)\right] .
$$

and the area of the horizon is

$$
\frac{A}{2 \pi^{2}}=\sqrt{\frac{1}{2}\left[3 q_{0}+\left(p^{1}\right)^{2}-\frac{16}{g^{2}}\right]\left[3 q_{1}+2 p^{1} p^{0}-\frac{8 p^{0}}{g}\right]\left[3 q_{1}+2 p^{0} p^{1}+\frac{8 p^{0}}{g}\right]-J^{2}} .
$$

\subsubsection{Null supersymmetric non-Abelian $5 d$ solutions from $4 d$ black holes and global monopoles}

Using the general results of the preceding sections it is very easy to construct null supersymmetric solutions by uplifting 4-dimensional timelike supersymmetric solutions with $\mathcal{I}^{0}$. In particular, we can uplift the black-hole and global-monopole solutions of the $\mathrm{ST}[2,5]$ model recently constructed in ref. [12]. In this paper we will focus on the single center solutions only.

The 4-dimensional solutions depend on the following non-vanishing $\mathcal{I}^{M}$

$$
\begin{aligned}
& \mathcal{I}^{1}=A^{1}+\frac{p^{1} / \sqrt{2}}{r}, \quad \mathcal{I}^{2}=A^{2}+\frac{p^{2} / \sqrt{2}}{r}, \quad \mathcal{I}^{A}=\sqrt{2} \delta^{A}{ }_{p} x^{p} f(r), \\
& \mathcal{I}_{0}=A_{0}+\frac{q_{0} / \sqrt{2}}{r},
\end{aligned}
$$

where $f(r)$ is the function $f_{\mu, s}$ or $f_{\lambda}$ in appendix $\mathrm{B}$ corresponding to one of the spherically-symmetric BPS SU(2) monopoles, $p^{1}, p^{2}, q_{0}$ are magnetic and electric charges and $A^{1}, A^{2}, A_{0}$ integration constants to be determined in terms of the asymptotic values of the scalars and the metric.

\footnotetext{
${ }^{32}$ We have not reexpressed the 4-dimensional gauge coupling constant $g$ in terms of the 5-dimensional, $\tilde{g}$ to have simpler expressions.
} 
The 5-dimensional metric is that of an intersection of a string lying along the $z$ direction and a $p p$-wave propagating along the same direction:

$$
d s^{2}=2 \ell d u(d v+K d u)-\ell^{-2} d \vec{x}_{(3)}^{2},
$$

where

$$
\ell^{-3}=4 \mathcal{I}^{1}\left[\left(\mathcal{I}^{2}\right)^{2}-2 r^{2} f^{2}\right], \quad K=1+2 \mathcal{I}_{0} .
$$

The scalar fields, defined by $\phi^{x} \equiv h^{x} / h^{0}$, are given by

$$
\phi^{1}=\mathcal{I}^{2} / \mathcal{I}^{1}, \quad \phi^{A}=-\delta^{A}{ }_{p} x^{p} f(r) / \mathcal{I}^{1},
$$

and the vector fields are given by

$$
A^{0,1}=-2 \sqrt{6} p^{1,2} A, \quad A^{A}=2 \sqrt{6} h(r) \epsilon^{A}{ }_{r s} x^{r} d x^{s},
$$

where $A$ is the vector field of a Dirac magnetic monopole of unit charge, satisfying $d A=\star_{3} d \frac{1}{r}$ and $h(r)$ is the function $h_{\mu, s}$ or $h_{\lambda}$ in appendix $\mathrm{B}$ corresponding to one of the spherically-symmetric BPS SU(2) monopoles.

The 4-dimensional electric charge $q_{0}$ corresponds to the momentum of the 5dimensional gravitational wave in the $z$ direction and none of the scalar and vector fields depend on it. For the sake of simplicity we are going to set it to zero $\left(q_{0}=0\right.$ and $\mathcal{I}_{0}=-1 / 2$ so $K=0$ ) and we are going to analyze the string solutions with the above scalar and vector fields and with metric

$$
d s^{2}=\ell\left(d t^{2}-d z^{2}\right)-\ell^{-2} d \vec{x}_{(3)}^{2},
$$

with the metric function $\ell$ given as above.

The metric will be regular in the $r \rightarrow 0$ limit if $\ell \sim r$ or $\ell \sim$ constant. These two behaviors are, respectively, those of extremal black strings in the near-horizon limit and those of global monopoles. Let us consider each case separately.

Global string-monopoles. These are the string-like solutions that, upon dimensional reduction along $z$, give the spherically-symmetric global monopoles constructed in ref. [12]. They can be constructed with $f(r)=f_{\mu, s=0}(r)$ (the BPST 't Hooft-Polyakov monopole) and with $p^{1}=p^{2}=0$, so that

$$
\ell^{-3}=4 A^{1}\left[\left(A^{2}\right)^{2}-2 r^{2} f_{\mu, s=0}^{2}\right], \quad \phi^{1}=A^{2} / A^{1}, \quad \phi^{A}=-\sqrt{2} \delta^{A}{ }_{r} x^{r} f_{\mu, s=0}(r) / A^{1},
$$

and the only non-trivial vector field is $A^{A}$.

The integration constants $A^{1,2}, \mu$ are given by

$$
A^{1}=\frac{1}{\chi_{\infty}^{1 / 3}}, \quad A^{2}=\frac{\phi_{\infty}^{1}}{\chi_{\infty}^{1 / 3}}, \quad \mu=\frac{g\left|\phi_{\infty}\right|}{\sqrt{2} \chi_{\infty}^{1 / 3}}, \quad \chi_{\infty} \equiv 4\left[\left(\phi_{\infty}^{1}\right)^{2}-\left|\phi_{\infty}\right|^{2}\right]
$$

where $\left|\phi_{\infty}\right|^{2}$ is the asymptotic value of the gauge-invariant combination $\phi^{A} \phi^{A}$, and the string's tension (simply defined as minus the coefficient of $1 / r$ in the large- $r$ expansion of $\left.g_{t t}\right)$ is given by $[29,30]$

$$
T_{\text {monopole }}=\frac{32\left|\phi_{\infty}\right|}{\sqrt{3} \chi_{\infty}^{2 / 3}} \frac{1}{|\tilde{g}|} .
$$


These are globally regular solutions with no horizons, like their 4-dimensional analogues.

Black strings. They must necessarily have non-vanishing magnetic charges $p^{1,2}$ in order to have a regular horizon. This horizon will be a 2-dimensional surface characterized by being normal to 2 linearly independent null vectors. The mass and entropy of the black string will depend on the choice of monopole.

Let us first consider the BPST 't Hooft-Polyakov monopole (or equivalently, let us add magnetic charges $p^{1,2}$ to the above global monopole). In this case, the relation between the integration constants $A^{1,2}, \mu$ and the asymptotic values of the scalars will be the same as before. The string's tension and the area of the horizon contain contributions from the magnetic charges $p^{1}, p^{2}$ :

$$
\begin{aligned}
T & =\frac{1}{3 \sqrt{2}} \chi_{\infty}^{1 / 3}\left[p^{1}+8 \frac{\phi_{\infty}^{1}}{\chi_{\infty}} p^{2}\right]+T_{\text {monopole }} \\
\frac{A}{4 \pi} & =2\left[p^{1}\left(p^{2}\right)^{2}\right]^{2 / 3}
\end{aligned}
$$

When we consider the more general 't Hooft-Polyakov-Protogenov monopole we find that the area of the horizon receives a contribution from the non-Abelian charge,

$$
\frac{A}{4 \pi}=2\left\{p^{1}\left[\left(p^{2}\right)^{2}-\frac{2}{g^{2}}\right]\right\}^{2 / 3} .
$$

\section{Conclusions}

In this paper we have studied the general procedure to construct timelike and null supersymmetric solutions of $\mathcal{N}=1, d=5$ SEYM theories that can be dimensionally reduced to timelike solutions of $\mathcal{N}=2, d=4$ SEYM theories. These solutions, therefore, can also be constructed by oxidation of the 4-dimensional solutions and we have striven to clarify this procedure and find the relations between the 4- and 5-dimensional fields and the 4and 5-dimensional equations they satisfy. The relation between instantons in 4-dimensional hyperKähler spaces and monopoles satisfying the Bogomol'nyi equation in $\mathbb{E}^{3}$ found by Kronheimer plays a crucial role in this relation and, in combination with the results obtained in ref. [4], it allows us to construct spherically-symmetric 5-dimensional solutions that contain YM instantons. The standard oxidation of monopoles gives rise to 5-dimensional solutions that have an additional translational isometry and cannot be spherically symmetric.

We have exploited the general results to construct the first 5-dimensional black-hole and black-string solutions with non-Abelian YM fields. The simplest black-hole solutions contain the field of a BPST instanton in the so-called base space and their behavior is similar to that of the colored black holes found in 4-dimensional SEYM theories [17, 25]: the non-Abelian YM field cannot be "seen" at spatial infinity, it does not contribute to the mass, but it can be seen in the near-horizon limit and it contributes to the entropy. One can compare the entropies of the simplest non-Abelian black hole with that of another black hole with the same Abelian charges and moduli (and, henceforth, with the same 
mass). The entropy of the former is always smaller, so it is entropically favorable to lose the non-Abelian field. It is not clear by which mechanism this can happen.

We have also found more complicated black-hole solutions which contain the field of the instantons that one obtains by oxidizing Protogenov monopoles in the so-called base space. Those instantons are not regular in flat space and, in general, the spacetime metrics they give rise to are not asymptotically flat. We have shown that a judicious choice of the integration constants (and, hence, of the moduli) in terms of the charges produces a metric that is not only asymptotically flat with positive mass but also has a regular horizon. Thus, at special points in the moduli space of the scalar manifold, additional non-Abelian black-hole solutions are possible. In these solutions, the YM fields do contribute to the mass and to the entropy.

Finally, we have also found black-string solutions by conventional oxidation of nonAbelian black-hole solutions from 4 dimensions. One of them is a globally-regular stringmonopole solution and the rest are more conventional solutions.

It is clear that the new solutions that we have constructed need further study. Their string-theoretic interpretation could be very interesting. The model we have chosen to construct explicit solutions is a truncation of the effective theory of the heterotic string compactified to 5 dimensions and can, alternatively, be seen as associated to the compactification of the type IIB theory in K3 times a circle. This should simplify a bit the task and, perhaps, open the way to a microscopic interpretation of entropies that depend on parameters that do not appear at infinity. Work in this direction is in progress.

\section{Acknowledgments}

The authors would like to thank A. Anabalón for interesting conversations. This work has been supported in part by the Spanish Ministry of Science and Education grants FPA201235043-C02 (-01 \& -02), the Centro de Excelencia Severo Ochoa Program grant SEV-20120249, the EU-COST Action MP1210 "The String Theory Universe", the Principado de Asturias grant GRUPIN14-108 and the Spanish Consolider-Ingenio 2010 program CPAN CSD2007-00042. The work was further supported by the Severo Ochoa pre-doctoral grant SVP-2013-067903 (PF-R). TO wishes to thank M.M. Fernández for her permanent support.

\section{A Dimensional reduction of $\mathcal{N}=1, d=5$ SEYM theories}

$\mathcal{N}=1, d=5$ supergravity coupled to vector multiplets gives $\mathcal{N}=2, d=4$ supergravity coupled to vector multiplets upon dimensional reduction over a spacelike circle. ${ }^{33}$ If some non-Abelian subgroup of the isometry group of the scalar manifold of the 5-dimensional theory has been gauged, and we perform a simple (as opposed to a generalized) dimensional reduction, the 4-dimensional theory will have exactly the same non-Abelian subgroup of the (now bigger) isometry group gauged. Thus $\mathcal{N}=1, d=5$ and $\mathcal{N}=2, d=4 \mathrm{SEYM}$ theories are related by dimensional reduction over a spacelike circle.

\footnotetext{
${ }^{33}$ See, for instance, refs. [31] and references therein.
} 
It should be clear that, under the above conditions, the relation between the 5 - and 4-dimensional fields in the gauged theories is exactly the same as in the ungauged one and is, therefore, well known. In the conventions we follow here ${ }^{34}$ the relation between the bosonic fields of an $\mathcal{N}=1, d=5$ supergravity model defined by $C_{I J K}$ (tilded) and the bosonic fields of a cubic model of $\mathcal{N}=2, d=4$ supergravity defined by the symmetric tensor $d_{i j k}$ (untilded) are $^{35}$

$$
\begin{array}{rlrl}
g_{\mu \nu} & =\left|\tilde{g}_{\underline{z z}}\right|^{\frac{1}{2}}\left(\tilde{g}_{\mu \nu}-\tilde{g}_{\mu \underline{z}} \tilde{g}_{\nu \underline{z}} / \tilde{g}_{\underline{z z}}\right), & d_{i j k}=6 C_{i-1 j-1 k-1}, \\
A^{0}{ }_{\mu}=\frac{1}{2 \sqrt{2}} \tilde{g}_{\mu \underline{z}} / \tilde{g}_{\underline{z z}}, & A_{\mu}^{i}=-\frac{1}{2 \sqrt{6}}\left(\tilde{A}_{\mu}^{i-1}-\tilde{A}_{\underline{\underline{z}}}^{i-1} \tilde{g}_{\mu \underline{z}} / \tilde{g}_{\underline{z z}}\right), \\
Z^{i}=\frac{1}{\sqrt{3}} \tilde{A}^{i-1}{ }_{\underline{z}}+i\left|\tilde{g}_{\underline{z z}}\right|^{\frac{1}{2}} \tilde{h}^{i-1}, & &
\end{array}
$$

and the inverse relations are

$$
\begin{aligned}
\tilde{g}_{z \underline{z}} & =-k^{2}, & \tilde{A}^{I}{ }_{\underline{z}} & =\sqrt{3} \Re \mathfrak{e} Z^{I+1}, \\
\tilde{g}_{\mu \underline{z}} & =-2 \sqrt{2} k^{2} A^{0}{ }_{\mu}, & \tilde{A}^{I}{ }_{\mu} & =-2 \sqrt{6}\left(A^{I+1}{ }_{\mu}-\Re \mathfrak{e} Z^{I+1} A^{0}{ }_{\mu}\right), \\
\tilde{g}_{\mu \nu} & =k^{-1} g_{\mu \nu}-8 k^{2} A^{0}{ }_{\mu} A^{0}{ }_{\nu}, & \tilde{h}^{I} & =k^{-1} \Im \mathfrak{m} Z^{I+1} .
\end{aligned}
$$

In these relations it has been taken into account that, if $n_{v}$ denotes the number of vector multiplets in $d=5$, then, the 4 -dimensional theory has $n_{v}+1$ vector multiplets so that $I, J, K=0, \cdots, n_{v}, i, j, k=0, \cdots, n_{v}+1$. The additional 4-dimensional vector multiplet is the $i=0$ one and, therefore, the 5 -dimensional vector labeled by $I$ corresponds to the 4-dimensional vector labeled by $i=I+1$.

While this is the whole story for the fields, it is important to realize that the factor that related the 4- and 5-dimensional gauge fields changes the standard form of the covariant derivatives and gauge field strengths and it must be absorbed into a redefinition of the gauge coupling constant. Thus, we also have

$$
\tilde{g}=-2 \sqrt{6} g .
$$

Observe that this result has been obtained using the orientation $\varepsilon^{0123 z}=+1$, which is not the one we are using in the main text $\left(\varepsilon^{0 z 123}=+1\right)$. However, in practice, the result can be adapted to that orientation by reversing the sign of each $z$ tensor index. This operation only changes the sign of $A^{0}{ }_{\mu}$ and $\Re \mathfrak{e} Z^{i}$.

\section{B Spherically-symmetric solutions of the SU(2) Bogomol'nyi equations in $\mathbb{E}^{3}$}

The equations of motion of the SU(2) Yang-Mills-Higgs (YMH) theory in the Bogomol'nyiPrasad-Sommerfield (BPS) limit in which the the Higgs potential vanishes read

$$
\mathfrak{D}_{\mu} F^{A \mu \nu}=-g \varepsilon_{B C}{ }^{A} \Phi^{B} \mathfrak{D}^{\nu} \Phi^{C}
$$

\footnotetext{
${ }^{34}$ That is, the conventions used in refs. $[1,14,19]$ for the $\mathcal{N}=1, d=5$ theories and in the conventions used in refs. $[2-4,12,13,17,25,32,33]$ for the $\mathcal{N}=2, d=4$ theories.

${ }^{35} \mathrm{See}$, for instance, ref. [7] which follows the conventions used here.
} 


$$
\mathfrak{D}^{2} \Phi^{A}=0 .
$$

Static configurations satisfying the first-order Bogomol'nyi equations [15]

$$
F_{\underline{r s}}^{A}=\varepsilon_{r s t} \mathfrak{D}_{\underline{t}} \Phi A,
$$

can be seen to satisfy all the above second-order YMH equations of motion.

BPS magnetic monopole solutions such as the (BPS) 't Hooft-Polyakov monopole found by Prasad and Sommerfield in ref. [34] satisfy the Bogomol'nyi equations and, therefore, it is of some interest to identify all their solutions. In the spherically-symmetric case this problem was solved by Protogenov in ref. [16] and his solution can be described as follows: the Higgs and gauge field can always be brought to this form (hedgehog ansatz)

$$
\Phi^{A}=-\delta^{A}{ }_{s} f(r) y^{s}, \quad A_{\underline{r}}^{A}=-\varepsilon_{r s}^{A} y^{s} h(r),
$$

in which they are characterized by just two functions, $f(r), h(r)$ of the radial coordinate $r=\sqrt{y^{s} y^{s}}$. There is only a 2-parameter family for which these functions, denoted by $\left(f_{\mu, s}, h_{\mu, s}\right)$, are given by

$$
r f_{\mu, s}=\frac{1}{g r}[1-\mu r \operatorname{coth}(\mu r+s)], \quad r h_{\mu, s}=\frac{1}{g r}\left[\frac{\mu r}{\sinh (\mu r+s)}-1\right],
$$

and a 1-parameter family for which these functions, denoted by $\left(f_{\lambda}, h_{\lambda}\right)$, are given by

$$
r f_{\lambda}=\frac{1}{g r}\left[\frac{1}{1+\lambda^{2} r}\right], \quad r h_{\lambda}=-r f_{\lambda} .
$$

The BPS 't Hooft-Polyakov monopole [34] is the only globally regular solution and corresponds to $f_{\mu, s=0}$. The $f_{\mu, s=\infty}$ solution is given by

$$
-r f_{\mu, \infty}=\frac{\mu}{g}-\frac{1}{g r}, \quad r h_{\mu, \infty}=-\frac{1}{g r},
$$

and, for $\mu=0$, it is the $\mathrm{Wu}$-Yang monopole [35]. The latter solution is also recovered in the 1-parameter family for $f_{\lambda=0}$.

The asymptotic behavior of $r f(r)$ (which is the combination that occurs in the metrics we study) for the different solutions is

$$
r f_{\mu, s} \sim-\frac{\mu}{g}+\frac{1}{g r}+\mathcal{O}\left(e^{-4 \mu r}\right), \quad-r f_{\lambda} \sim \frac{1}{g \lambda^{2} r^{2}}+\mathcal{O}\left(r^{-3}\right),
$$

and the behavior near the origin (where the black-hole horizons may be in the metrics under study) are

$$
r f_{\mu, 0} \sim-\frac{\mu^{2}}{2 g} r+\mathcal{O}\left(r^{3}\right), \quad r f_{\mu, s} \sim \frac{1}{g r}-\frac{\mu}{g} \operatorname{coth} s+\mathcal{O}(r), \quad r f_{\lambda} \sim \frac{1}{g r}-\frac{\lambda^{2}}{g} r+\mathcal{O}\left(r^{3}\right) .
$$

If we define the magnetic monopole charge by

$$
p \equiv \frac{1}{4 \pi} \int_{S_{\infty}^{2}} \operatorname{Tr}(\hat{\Phi} F), \quad \hat{\Phi} \equiv \frac{\Phi}{\sqrt{\left|\operatorname{Tr}\left(\Phi^{2}\right)\right|}},
$$

then, we always find $p=1 / g$ except in the 1-parameter family for finite $\lambda$, for which we find $p=0$. As we have argued in ref. [12], the $\lambda \neq 0$ colored monopoles can be seen as a magnetic monopole placed at the origin whose charge is completely screened at infinity. 
Open Access. This article is distributed under the terms of the Creative Commons Attribution License (CC-BY 4.0), which permits any use, distribution and reproduction in any medium, provided the original author(s) and source are credited.

\section{References}

[1] J. Bellorín and T. Ortín, Characterization of all the supersymmetric solutions of gauged $N=1, D=5$ supergravity, JHEP 08 (2007) 096 [arXiv:0705.2567] [INSPIRE].

[2] M. Hübscher, P. Meessen, T. Ortín and S. Vaulà, Supersymmetric $N=2$ Einstein-Yang-Mills monopoles and covariant attractors, Phys. Rev. D 78 (2008) 065031 [arXiv:0712.1530] [INSPIRE].

[3] M. Hübscher, P. Meessen, T. Ortín and S. Vaulà, $N=2$ Einstein-Yang-Mills's BPS solutions, JHEP 09 (2008) 099 [arXiv:0806.1477] [INSPIRE].

[4] P. Bueno, P. Meessen, T. Ortín and P.F. Ramírez, Resolution of SU(2) monopole singularities by oxidation, Phys. Lett. B 746 (2015) 109 [arXiv: 1503. 01044] [INSPIRE].

[5] R. Bartnik and J. Mckinnon, Particle-like solutions of the Einstein Yang-Mills equations, Phys. Rev. Lett. 61 (1988) 141 [INSPIRE].

[6] M.S. Volkov and D.V. Galtsov, Non-Abelian Einstein Yang-Mills black holes, JETP Lett. 50 (1989) 346 [Pisma Zh. Eksp. Teor. Fiz. 50 (1989) 312] [INSPIRE].

[7] T. Ortín, Gravity and strings, $2^{\text {nd }}$ edition, Cambridge University Press, Cambridge U.K. (2015).

[8] J.A. Harvey and J. Liu, Magnetic monopoles in $N=4$ supersymmetric low-energy superstring theory, Phys. Lett. B 268 (1991) 40 [INSPIRE].

[9] A.H. Chamseddine and M.S. Volkov, Non-Abelian BPS monopoles in $N=4$ gauged supergravity, Phys. Rev. Lett. 79 (1997) 3343 [hep-th/9707176] [INSPIRE].

[10] A.H. Chamseddine and M.S. Volkov, Non-Abelian solitons in $N=4$ gauged supergravity and leading order string theory, Phys. Rev. D 57 (1998) 6242 [hep-th/9711181] [INSPIRE].

[11] R. Kallosh and T. Ortín, Exact $\mathrm{SU}(2) \times \mathrm{U}(1)$ stringy black holes, Phys. Rev. D 50 (1994) 7123 [hep-th/9409060] [INSPIRE].

[12] P. Bueno, P. Meessen, T. Ortín and P.F. Ramirez, $N=2$ Einstein-Yang-Mills'static two-center solutions, JHEP 12 (2014) 093 [arXiv:1410.4160] [INSPIRE].

[13] P. Meessen and T. Ortín, Supersymmetric solutions to gauged $N=2 D=4$ SUGRA: the full timelike shebang, Nucl. Phys. B 863 (2012) 65 [arXiv:1204.0493] [INSPIRE].

[14] J. Bellorín, Supersymmetric solutions of gauged five-dimensional supergravity with general matter couplings, Class. Quant. Grav. 26 (2009) 195012 [arXiv:0810.0527] [INSPIRE].

[15] E.B. Bogomolny, Stability of classical solutions, Sov. J. Nucl. Phys. 24 (1976) 449 [Yad. Fiz. 24 (1976) 861] [INSPIRE].

[16] A.P. Protogenov, Exact classical solutions of Yang-Mills sourceless equations, Phys. Lett. B 67 (1977) 62 [INSPIRE].

[17] P. Meessen, Supersymmetric coloured/hairy black holes, Phys. Lett. B 665 (2008) 388 [arXiv: 0803.0684] [INSPIRE]. 
[18] P.B. Kronheimer, Monopoles and Taub-NUT spaces, M.Sc. dissertation, Oxford University, Oxford U.K. (1995).

[19] J. Bellorín, P. Meessen and T. Ortín, All the supersymmetric solutions of $N=1, d=5$ ungauged supergravity, JHEP 01 (2007) 020 [hep-th/0610196] [INSPIRE].

[20] E. Bergshoeff, S. Cucu, T. de Wit, J. Gheerardyn, S. Vandoren and A. Van Proeyen, $N=2$ supergravity in five-dimensions revisited, Class. Quant. Grav. 21 (2004) 3015 [Corrigendum ibid. 23 (2006) 7149] [hep-th/0403045] [INSPIRE].

[21] J.P. Gauntlett, J.B. Gutowski, C.M. Hull, S. Pakis and H.S. Reall, All supersymmetric solutions of minimal supergravity in five-dimensions, Class. Quant. Grav. 20 (2003) 4587 [hep-th/0209114] [INSPIRE].

[22] J.P. Gauntlett and J.B. Gutowski, General concentric black rings, Phys. Rev. D 71 (2005) 045002 [hep-th/0408122] [INSPIRE].

[23] G.W. Gibbons and P.J. Ruback, The hidden symmetries of multicenter metrics, Commun. Math. Phys. 115 (1988) 267 [INSPIRE].

[24] G.W. Gibbons and S.W. Hawking, Gravitational multi-instantons, Phys. Lett. B 78 (1978) 430 [InSPIRE].

[25] P. Meessen and T. Ortín, $N=2$ super-EYM coloured black holes from defective Lax matrices, JHEP 04 (2015) 100 [arXiv:1501.02078] [INSPIRE].

[26] C.A.R. Herdeiro, Spinning deformations of the D1-D5 system and a geometric resolution of closed timelike curves, Nucl. Phys. B 665 (2003) 189 [hep-th/0212002] [INSPIRE].

[27] J.C. Breckenridge, R.C. Myers, A.W. Peet and C. Vafa, D-branes and spinning black holes, Phys. Lett. B 391 (1997) 93 [hep-th/9602065] [INSPIRE].

[28] J.P. Gauntlett, R.C. Myers and P.K. Townsend, Black holes of D $=5$ supergravity, Class. Quant. Grav. 16 (1999) 1 [hep-th/9810204] [INSPIRE].

[29] A. de Antonio Martin, T. Ortín and C.S. Shahbazi, The FGK formalism for black p-branes in d dimensions, JHEP 05 (2012) 045 [arXiv: 1203.0260] [INSPIRE].

[30] P. Meessen, T. Ortín, J. Perz and C.S. Shahbazi, Black holes and black strings of $N=2$, $D=5$ supergravity in the H-FGK formalism, JHEP 09 (2012) 001 [arXiv: 1204.0507] [INSPIRE].

[31] D.Z. Freedman and A. Van Proeyen, Supergravity, Cambridge Univ. Pr., Cambridge U.K. (2012).

[32] P. Meessen and T. Ortín, The supersymmetric configurations of $N=2, D=4$ supergravity coupled to vector supermultiplets, Nucl. Phys. B 749 (2006) 291 [hep-th/0603099] [INSPIRE].

[33] M. Hübscher, P. Meessen and T. Ortín, Supersymmetric solutions of $N=2 D=4$ SUGRA: the whole ungauged shebang, Nucl. Phys. B 759 (2006) 228 [hep-th/0606281] [InSPIRE].

[34] M.K. Prasad and C.M. Sommerfield, An exact classical solution for the 't Hooft monopole and the Julia-Zee dyon, Phys. Rev. Lett. 35 (1975) 760 [InSPIRE].

[35] T.T. Wu and C.-N. Yang, Some solutions of the classical isotopic gauge field equations, in Selected papers 1945-1980, C.N. Yang, pg. 400 also in Properties of matter under unusual conditions, H. Mark and S. Fernbach, New York U.S.A. (1969), pg. 349. 